\title{
Can Computing Be Diversified on "Principles" Alone? Exploring the Role of AP Computer Science Courses in Students' Major and Career Intentions
}

\author{
LINDA J. SAX, University of California, Los Angeles \\ KAITLIN N. S. NEWHOUSE, North Carolina State University \\ JOANNA GOODE, University of Oregon \\ TOMOKO M. NAKAJIMA, University of California, Los Angeles \\ MAX SKORODINSKY, University of Oregon \\ MICHELLE SENDOWSKI, University of California, Los Angeles
}

\begin{abstract}
The Advanced Placement Computer Science Principles (APCSP) course was introduced in 2016 to address long-standing gender and racial/ethnic disparities in the United States among students taking Advanced Placement Computer Science (APCS) in high school, as well as among those who pursued computing majors in college. Although APCSP has drawn a more diverse population of course takers than the traditional Advanced Placement Computer Science A (APCSA) course, questions remain about whether the new course portends students' longer-term interest in computing and technology. This study used data from more than 120,000 first-year college students in the United States to understand the relationship between taking APCSA, APCSP, or both APCS courses and students' aspirations to major in computing or to pursue a computing or technology career. Our results indicated that taking APCSA or taking both APCS courses positively predicted students' major or career aspirations in computing and technology, but that taking only APCSP did not predict long-term computing interest (although positive associations were identified for women). The findings identify a possible tension in the function of APCSP, as it appears to broaden access to computing knowledge, but may not necessarily encourage longer-term computing aspirations for all groups of underrepresented students.
\end{abstract}

CCS Concepts: $\bullet$ Social and professional topics $\rightarrow$ Professional topics; Computing education; Computing education programs; Computer science education;

Additional Key Words and Phrases: AP Computer Science A course/exam, AP Computer Science Principles course/exam, gender and race/ethnicity

Authors' addresses: L. J. Sax (corresponding author), School of Education and Information Studies, University of California, Los Angeles, 3045 Moore Hall, Mailbox 951521, Los Angeles, CA 90095-1521; email: lsax@g.ucla.edu; K. N. S. Newhouse, Belk Center for Community College Leadership and Research at NC State University, 706 Hillsborough Street, Raleigh, NC, 27603; email: knnewhou@ncsu.edu; J. Goode, College of Education, 1215 University of Oregon Eugene, OR 97403-1215; email: goodej@uoregon.edu; T. M. Nakajima, USchool of Education and Information Studies, University of California, Los Angeles, 405 Hilgard Avenue Los Angeles, California 90095-1521; email: tnak493@gmail.com; M. Skorodinsky, College of Education 1215 University of Oregon Eugene, OR 97403-1215; email: makseem@uoregon.edu; M. Sendowski, School of Education and Information Studies, University of California, Los Angeles, 405 Hilgard Avenue Los Angeles, California 90095-1521; email: michelle.sendowski@gmail.com.

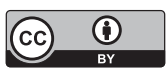

This work is licensed under a Creative Commons Attribution International 4.0 License.

(C) 2022 Copyright held by the owner/author(s).

1946-6226/2022/02-ART18

https://doi.org/10.1145/3479431 
ACM Reference format:

Linda J. Sax, Kaitlin N. S. Newhouse, Joanna Goode, Tomoko M. Nakajima, Max Skorodinsky, and Michelle Sendowski. 2022. Can Computing Be Diversified on "Principles" Alone? Exploring the Role of AP Computer Science Courses in Students' Major and Career Intentions. ACM Trans. Comput. Educ. 22, 2, Article 18 (February 2022), 26 pages.

https://doi.org/10.1145/3479431

\section{INTRODUCTION}

Computer science (CS) holds immense power and influence in our global society and economy, and yet from its origins has been and remains an elite and exclusionary field. Indeed, despite recent concerted efforts in the United States to broaden participation in the field, the demographics of the innovators and creators of technology still do not reflect the racial, cultural, and gender diversity of our society [28]. Exemplifying this imbalance, women represent $57 \%$ of the total U.S. workforce but constitute only $26 \%$ of the computing workforce [45], and although Black and Hispanic workers constitute $11 \%$ and $16 \%$ of the total workforce, respectively, each group represents only $7 \%$ of the computing workforce [22].

Disparities in computing participation begin early in school settings, as the literacies and experiences shown to motivate youth to enroll in introductory CS courses are often acquired through out-of-school programs predominantly accessed by students from affluent families [3,29]. For people from historically minoritized groups to benefit from participating in and shaping the computing field, and for the computing field to reflect and benefit from the rich diversity of the larger society, a broader profile of youth must consider studying CS in higher education, and computing's distinction as an elite form of knowledge must be dismantled at the elementary and secondary school levels [1,39]. A key focus of such efforts to broaden participation in computing (BPC) has been to expand CS education opportunities in the United States. In the past 10 years, a series of policy reforms and industry-backed investments have steadily increased access to CS learning opportunities in working-class neighborhoods in the United States through after-school programs [38], summer coding camps [40], and library makerspaces [9], and through the introduction of computing courses like Exploring Computer Science in high schools across the country [24, 44].

In this article, we examine another BPC initiative, the relatively new Advanced Placement (AP) course that launched in 2016: Advanced Placement Computer Science Principles (APCSP). APCSP was designed specifically to increase interest in CS among a more diverse population of high school students and to expand the number of students prepared to meet the labor demands of the exponentially growing computing workforce $[18,68]$. We compare three groups of students-those who took the new APCSP course, those who took the legacy Advanced Placement Computer Science A (APCSA) course, and students who took both Advanced Placement Computer Science (APCS) courses-to examine the extent to which these courses portend undergraduate students' intentions to major in or pursue careers in computing.

\section{REVIEW OF THE LITERATURE}

\subsection{The School-Career Computing "Pipeline"}

The pipeline metaphor is frequently used to explain demographic disparities in science, technology, engineering, and math (STEM) education and the workforce, as it demonstrates how an essential series of experiences and knowledge starting in childhood are necessary for entry into certain elite professions [43]. The pipeline analogy is particularly pervasive in CS education research [59], given the tendency to view computing education as the key to computing majors and jobs $[3,29]$. Thus, based on the pipeline metaphor, persistent demographic disparities in the field 
can be attributed in part to the fact that affluent white male students simply take more preparatory CS courses progressively through high school and beyond [30, 46].

However, the computing career pipeline metaphor has been critiqued for ignoring the broader systemic forces that perpetually limit opportunities for students to access the coursework and social capital necessary to enter and remain in the computing pipeline [44, 59, 66]. Further, the metaphor fails to explain why the gender and racial/ethnic disparities prevalent in computing majors and careers in the United States, Canada, and Western Europe are not observed elsewhere in the world [32]. Accordingly, programmatic efforts to address diversity in STEM careers using pipeline metaphors have been criticized for forwarding a westernized neoliberal paradigm of education and failing to acknowledge the problematic and undemocratic nature of science itself [42]. Indeed, when it is assumed that there is a single pathway to computing majors and jobs, touchstones like APCS courses can act as gatekeepers that exclude individuals from entering the pipeline, disproportionately affecting students impacted by a nexus of systemic oppressions like racism, sexism, and classism, who are least likely to have access to such courses [66].

A long-standing offering in the computing pipeline is APCSA, a high school elective first offered in 1984. With a focus on Java programming, APCSA was designed to be comparable to a collegelevel introductory class for CS majors and is considered a valuable gateway course for high school students in the United States; it is closely associated with career readiness and interest in pursuing employment in a computing field [3, 29]. A longitudinal study revealed that $18 \%$ of APCSA coursetakers eventually majored in a computing-related domain in college, a rate eight times higher than AP students of other courses [41]. In addition, grades earned in AP classes can augment a coursetaker's high school grade point average (GPA), and a passing score on the end-of-course AP exam is often exchanged for undergraduate credit and higher placement in the CS course sequence in college [15].

Despite the role that early CS course-taking plays in shaping subsequent pursuit of CS, scholars have found that computing courses are taught at only about half of the high schools across the United States and that, among the classes that are offered, nearly half do not actually introduce students to programming concepts [5]. In fact, although AP courses are available to students across the globe through in-person schools and online programs, APCSA, like all AP courses, is offered more consistently at high schools in affluent neighborhoods in the United States [5, 58, 63], where educators explicitly steer many more students toward computing opportunities [23, 39]. In part because of this inequitable access to courses across schools, 78\% of APCSA exam-takers identify as white or Asian [68]. To summarize, although students without access to AP classes are already disadvantaged when applying to college [63], taking and passing APCSA gives other students a competitive edge and accelerates individuals toward related college majors and careers in CS in the United States and at the more than 400 institutions outside the United States that use AP courses in their admissions and course-placement procedures [14].

\subsection{APCSP to Broaden Participation in Computing}

In the aforementioned historic movement of sweeping national BPC efforts in the United Statesand with significant support from the National Science Foundation and unprecedented influence from the technology industry and industry-backed non-profit partners-the College Board, which administers the AP program, designed a new introductory CS course. APCSP was conceived to address the long-standing racial, gender, and socioeconomic disparities represented in APCSA, in higher education, and in computing careers in the United States [3]. College Board president and CEO David Coleman articulated that the purpose of APCSP was to "cultivate an interest in CS among students of all backgrounds" and to prepare course-takers for "the computing jobs that will 
help power future economic growth" [25]. In other words, he explicated before the course launched that two key goals of APCSP were to diversify the group of students who studied computing in high school (expand access to the pipeline) and increase the number of trained and qualified people who will eventually work in computing (broaden the pipeline).

To "motivate and educate a substantially larger number of students to fulfill the demands of the market while reaching a broader, more diverse segment of the population" [2], APCSP course content emphasizes the big intellectual and fundamental ideas of computing. The focus on foundational concepts in computing such as creativity, abstraction, data and information, algorithms, programming, the Internet, and the global impact of computing-rather than APCSA's narrower focus on programming-was designed in part to better attract and retain students from groups that are underrepresented in computing, including girls, people of color, and persons with disabilities [18]. Further, unlike APCSA, which assesses student learning with a summative final AP exam, a distinct aspect of the APCSP assessment is the inclusion of an applied performance task, centering choice and creativity in students' computational work, which is created during classroom instructional time and ultimately accounts for $40 \%$ of students' final AP score.

2.2.1 The Launch of APCSP. In fall 2016, APCSP had the largest launch of any AP course in the College Board's history [15]. In its inaugural year, 43,780 students from 2,500 schools took the APCSP exam [16]; by 2018, this figure had more than doubled to 94,360 . Headlines from organizations that promoted diversity initiatives in computing touted victory, stating: "Girls and minorities break records in computer science as fastest-growing groups . . . thanks to the recordbreaking launch of the new Computer Science Principles" [12]. To be sure, recent data suggests that APCSP attracts a far more diverse group of students in terms of gender and race/ethnicity than does the traditional APCSA course [55, 68], although participation among girls and Black and Hispanic students still does not mirror the diversity of the nation's high school student body.

Although there is evidence that APCSP contributes to both expanding and diversifying the pool of high school students learning college-level CS, there is more to explore about what role APCSP plays in ultimately diversifying computing spaces in higher education and the workforce. Evidence from the College Board [68] suggests that the introduction of APCSP is correlated with a growing number of students majoring in CS in college, revealing that students who take APCSP are more likely to major in CS in college than similar students who graduated high school just prior to the introduction of APCSP (16.9\% in 2019 vs. $5.2 \%$ in 2016). Their analysis matched students graduating high school in 2019 with those graduating in 2016 in terms of gender, race/ethnicity, parental education, high school GPA, and SAT; however, it did not account for additional variables that may correlate with enrollment in APCS courses or otherwise predict major choice, which is a goal of the present study.

\section{OBJECTIVES}

This empirical inquiry builds on prior studies of college students who had participated in one or both APCS courses [26, 57, 68] to examine associations between taking APCSA, APCSP, or both APCS courses and students' aspirations to pursue majors or careers in computing. The study includes a set of control variables similar to that used in the College Board [68] study (e.g., gender, race/ethnicity, parental education, high school GPA, and standardized test scores), in addition to a wide range of other covariates associated with major and career decision-making. Specifically, using 2017 data from The Freshman Survey (TFS), a nationwide survey of first-year college students administered the year following the formal launch of APCSP, we examined the following research questions: 
(1) How does taking APCSA, APCSP, or both APCS courses correspond with students' intent to major in a computing field or aspire to a career in computing, and how does this vary by students' gender or race/ethnicity?

(2) Controlling for proxies for academic achievement (e.g., high school GPA, SAT/ACT scores), to what extent does APCS course-taking predict students' intent to major in a computing field or aspire to a career in computing?

(a) Are there differences in the predictive power of taking only APCSP, only APCSA, or both APCS courses?

(b) Does the predictive power of taking each course differ by students' gender or race/ethnicity?

\section{METHODS}

\subsection{Data Source and Sample}

Data for this study were provided by UCLA's Higher Education Research Institute (HERI). Each year, HERI administers TFS to first-year college students attending institutions that participate in the Cooperative Institutional Research Program. Administered annually since 1966, this comprehensive survey asks respondents about their demographic characteristics, values and beliefs, academic and social involvement during their last year of high school, and their expectations and goals for their college experience and career. This study relied on data from the 2017 TFS, as the APCSP course officially launched in fall 2016 (with a more limited set of schools piloting the course and exam in the 2 years prior) [19]. Thus, these TFS respondents from 168 four-year colleges and universities [64 $]^{1}$ are the first class of students who would have had the opportunity to take the fully approved and more widely offered APCSP course. Among the more than 129,000 respondents in our sample are 6,098 students who took only APCSA, 1,851 who took only APCSP, and 896 who took both APCS courses. Demographically, $58.6 \%$ of respondents from the full TFS sample indicated they were women, and the racial/ethnic distribution of the sample was as follows: $0.2 \%$ were American Indian, $14.5 \%$ were Asian or Asian American, $8.7 \%$ were Black or African American, 8.7\% were Hispanic or Latinx, 55.3\% were white, $11.9 \%$ indicated two or more races/ethnicities, and $0.8 \%$ were some other race/ethnicity.

\subsection{Social Cognitive Career Theory}

We employed social cognitive career theory (SCCT) [34] as a framework to guide our investigation of the relationships between APCS course-taking and intentions to pursue a computing major or career. Based on Bandura's social cognitive theory [4], SCCT models how an individual's personal characteristics (e.g., race/ethnicity, gender, academic achievement) and their learning experiences might interact with their self-efficacy beliefs, expectations, interests, and goals, to ultimately shape their academic and career aspirations [34-36]. The model accounts for a wide variety of student identities and conditions that impact students' career development and has been found to be helpful for framing educational and career development in STEM fields broadly [11, 62, 67] and in specific fields like computing [33, 57] and engineering [27, 31]. Additionally, scholars have used SCCT to explore how secondary and postsecondary schools might increase the participation of students historically marginalized in STEM education [21, 47, 53, 65]. Our study builds on this prior research by using SCCT to investigate the potential of APCS course-taking in increasing the racial/ethnic and gender diversity in computing and technology, which is a stated goal of the APCSP course [19]. See Figure 1 for our adaptation of the model.

\footnotetext{
${ }^{1}$ Detailed demographic characteristics of APCS course-takers among the 2017 TFS respondents can be found in our earlier work [57].
} 


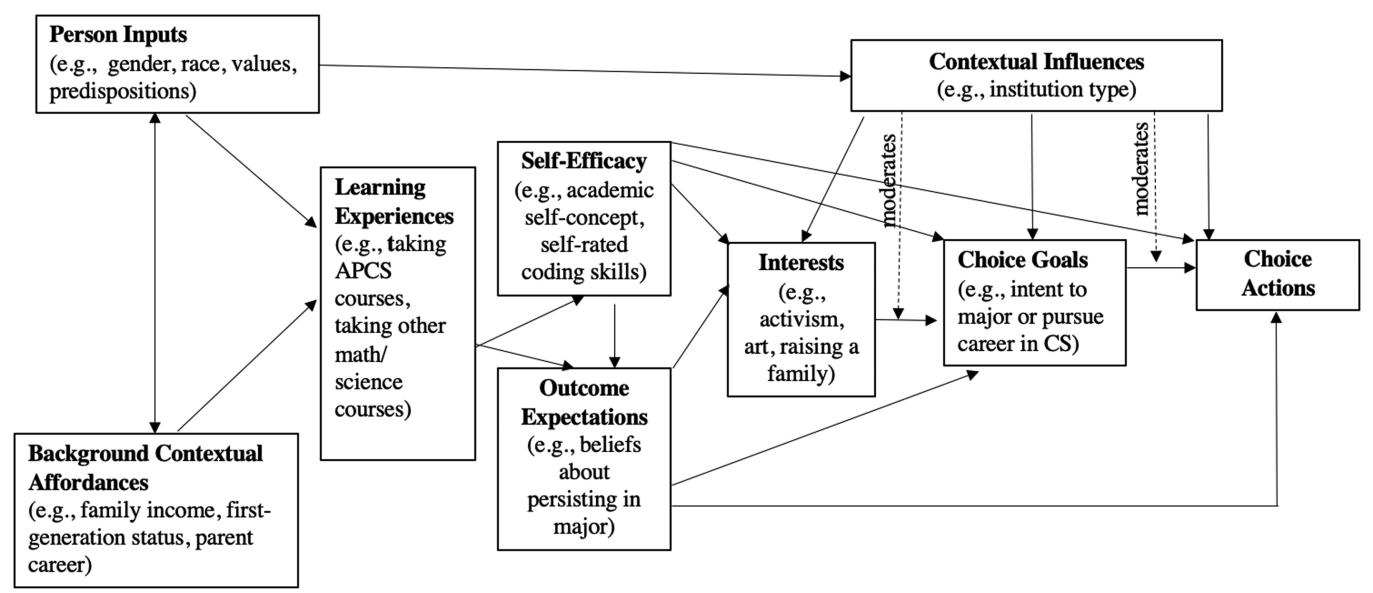

Fig. 1. SCCT model. Adapted from Lent et al. [34].

We applied SCCT to control for a wide variety of experiences, beliefs, and interests that inform two different choice goals, defined as the "intentions, plans, or aspirations to engage in a particular career direction" [34] among undergraduate students: (1) their intent to major in computing and (2) their aspirations for a career in computing/technology. However, for the purposes of this study, we were most interested in the role of particular learning experiences: having taken APCSA, APCSP, or both APCS courses. The SCCT framework suggests that person inputs (e.g., race/ethnicity, gender) and background contextual affordances (e.g., family income, parent education) often dictate students' access to and perceptions of learning experiences, and it is such learning experiences (like APCS courses) that inform individuals' self-efficacy, or beliefs about how well one will execute tasks or be successful in a specific domain [34]. Thus, the model suggests that the greater an individual's self-efficacy in a task like computer programming, for example, the more likely they will be to have more positive outcome expectations of majoring or working in a computing field successfully. Self-efficacy and outcome expectations also influence students' interests or enjoyment in participating in activities, especially those related to the choice goal in question [34]. Consequently, these interests inform students' choice goals. Finally, the framework accounts for the fact that students develop interests, goals, and aspirations about their careers amidst particular contextual influences.

\subsection{Measures}

Our logistic regression analyses (described in the following) relied on two dependent variables: (1) students' intent to major in computing and (2) students' intent to work in a computing or technology profession. The computing major variable was created by aggregating 90 options for academic major into a dichotomous measure for intended computing major. Students were considered computing majors if they indicated their probable major was "computer science," "computer/management information systems," or "other math/computer science." If they listed any other major, they were considered a non-computing major. Similarly, the dependent variable measuring career interest in computing and technology aggregated the 64 career options on the survey into a dichotomous variable that represented students' interest either in a career in computing and technology (which included "computer programmer/developer," "computer systems analyst," or "web designer") or an interest in any other career.

Independent variables for both regressions were selected and blocked based on SCCT [34-36] (see Appendix Table A.1). The person inputs block included both personal traits and values, and 
included self-reported social identities (gender ${ }^{2}$, race/ethnicity ${ }^{3}$, first-generation status), as well as students' belief that programming is important for most careers. The block representing background contextual affordances controlled for additional factors that might influence students' goals and beliefs [34], and included measures of family income, parent education, and whether or not students had at least one parent in a STEM profession. The learning experiences block consisted of measures of academic achievement in high school, such as GPA, SAT/ACT score(s), and time spent writing computer code. This block also included our key independent variables of interest: the three dichotomous measures indicating whether students self-reported having taken APCSA, APCSP, or both APCS courses.

The self-efficacy block included measures of students' confidence in their computer programming skills, as well as constructs measuring their academic self-concept, science self-efficacy, and science identity, among others. The outcome expectations block captured students' beliefs about what might happen should they make certain major or career choices, and it was the only block that differed between the models. In the regression predicting students' probable major, we used students' confidence that they would remain in their current major of interest, whereas the model examining students' career aspirations included a measure representing students' confidence that they would maintain their current career plans.

Variables in the interests block included measures representing students' reasons for attending college, such as to make more money or to gain an appreciation of ideas. We also used a construct representing students' social agency, or the degree to which students are invested in the social welfare of others, as well as measures indicating students' interest in raising a family, identification as artists, or as goal-driven strivers. The contextual influences block included institutional characteristics, such as a measure of institutional selectivity and whether the college is public or private. Finally, we also controlled for students' educational degree aspirations. In sum, SCCT guided our selection of independent variables and enabled us to examine the predictive power of APCS courses in light of variables understood to predict major and career choice, thus distinguishing this study from related research conducted by the College Board [68].

4.3.1 Constructs and Factor Analysis. To reduce the number of observed variables and to capture unobserved latent constructs, we performed exploratory factor analysis using principal axis factoring with Promax rotation. These analyses were based on factors used in prior research on undergraduate computing major choice [56] and the impact of college on students [54]. All factors met the reliability thresholds of a Cronbach's alpha of .65 or higher, and items had individual factor loadings greater than .4. We also made use of existing CIRP constructs developed and validated by researchers at the Higher Education Research Institute [17]. These constructs were developed using item response theory, and thus we do not report Cronbach's alpha or factor loadings for these items [60]. Further descriptions of factors and constructs and the individual items therein can be found in Appendix Table A.1.

4.3.2 Missing Data. We used the multiple imputation procedures in SPSS 25 to address missing data. Multiple imputation is currently the most robust method when data is not missing at random [10]. The procedure involved making a predetermined number of imputations or estimates

\footnotetext{
${ }^{2}$ The TFS asks students to identify as either male or female. In this article, we refer to respondents as men or women as an indication of gender identity rather than biological sex, despite the fact that this variable likely does not capture all students who identify as men or women or any gender identities beyond this binary.

${ }^{3}$ The TFS aggregates students' self-selected racial identities into seven broad categories (shown in Appendix A). We recognize that these groupings may reveal racial/ethnic trends in our data while simultaneously obfuscating any heterogeneity that exists within each group and among individuals who do not identify with any of the racial/ethnic groups named in the survey.
} 


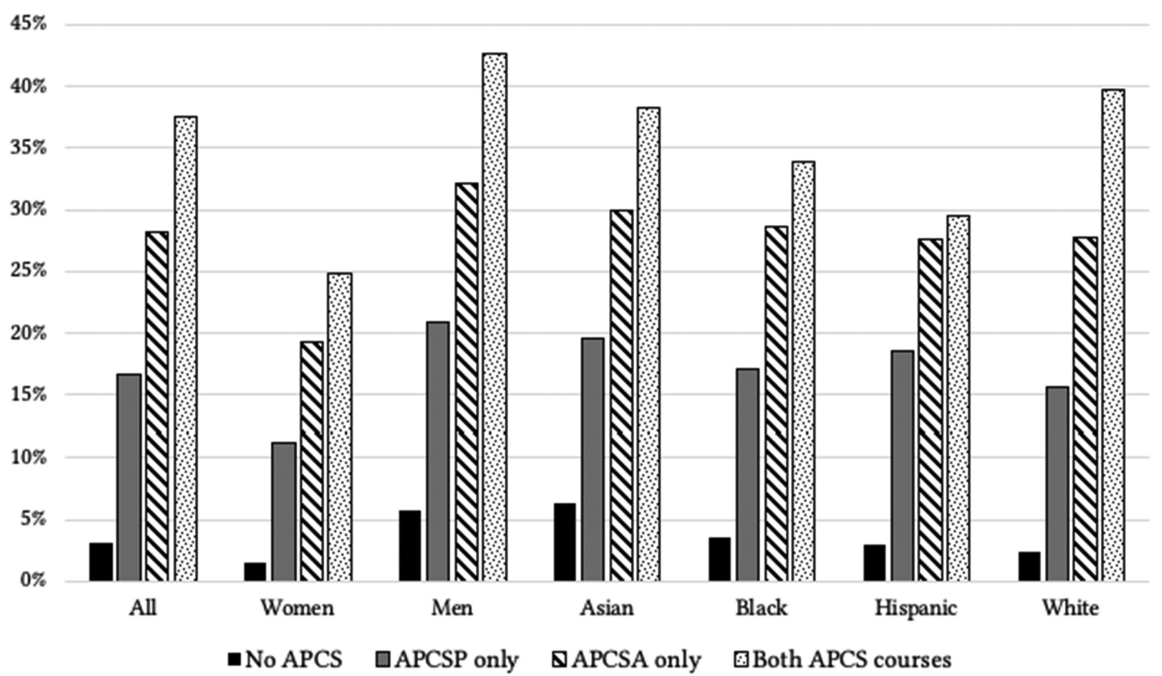

Fig. 2. Percentage of first-year undergraduate sample planning to major in computing, by gender, race, and AP course-taking, fall 2017.

for each missing value based on existing values in the dataset, then pooling all of the estimates to account for bias in the estimates. Demographic characteristics and our dependent variables were not imputed but were included in imputation models to aid in predicting values of other missing variables. Results reported in Tables 1 and 2 are based on the pooled estimates across 50 imputations. Although there is currently no way to pool estimates of commonly reported fit indices for logistic regression (e.g., pseudo R-squared, Hosmer-Lemeshow), indices for all 50 imputations are available upon request.

\subsection{Descriptive Analysis}

To address our first research question, cross tabulations were run to compare the percentage of students who indicated interest in pursuing computing majors or careers as it varied by whether they took APCSA only, APCSP only, both APCS courses, or neither APCS course. This analysis was conducted separately by gender and race/ethnicity (Figures 2 and 3).

\subsection{Regression Analysis}

To address our second research question and its subparts, we ran one logistic regression to estimate the relationship between APCS course-taking and first-year college students' interest in majoring in computing (see Table 1) and another logistic regression to explore students' career aspirations (see Table 2). For each regression, we first ran a main effects model (Model 1) that controlled for a variety of possible determinants and influences on the dependent variables. We then ran a model that included the main effects and 18 interaction terms (i.e., each of the five race/ethnicity variables by the three AP course variables; gender by the three AP course variables) (Model 2).

\section{RESULTS}

\subsection{Research Question 1: Descriptive Results}

Our first research question focused on how taking APCSA, APCSP, or both APCS courses corresponds with entering college students' interest in pursuing computing majors and careers across 


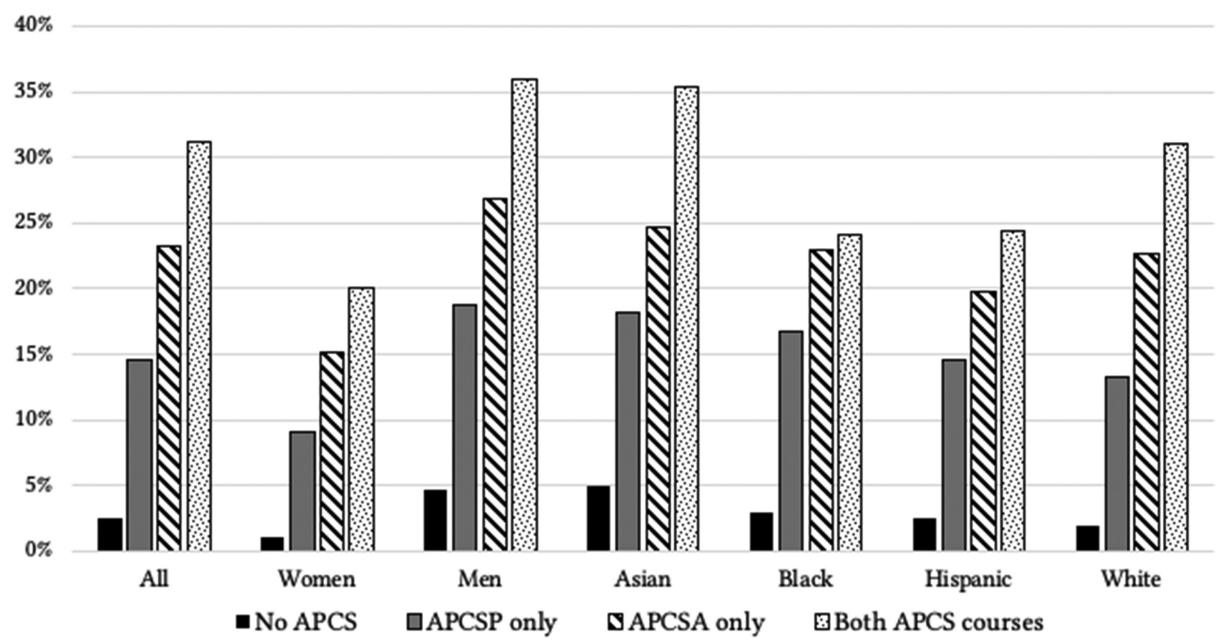

Fig. 3. Percentage of first-year undergraduate sample planning to pursue a career in computing, by gender, race, and AP course-taking, fall 2017.

categories of gender and race/ethnicity. As shown in Figure 2, interest in pursuing a computing major among all students was highest among those who took both APCS courses (37.5\%), followed by students who took APCSA only (28.2\%), those who took APCSP only (16.7\%), and those who took neither APCS course (3.1\%). This general pattern held across gender and racial/ethnic groups.

A similar pattern emerged with respect to interest in a computing career (see Figure 3), as interest among all students was highest among those who took both APCS courses (31.2\%), followed by those who took APCSA only (23.2\%), those who took APCSP only (14.5\%), and those who took neither APCS course (2.5\%). Once again, this pattern generally held across gender and racial/ethnic identities.

\subsection{Research Question 2: Regression Results}

The results presented thus far reveal, not surprisingly, a higher degree of interest in computing majors and careers among students who took APCS courses, with even more interest among those who took APCSA only or both APCS courses. An important question is whether these associations hold once we accounted for the full range of independent variables included in this study. As such, we now turn to research question 2 .

In presenting these results, we focus primarily on how the three APCS variables predicted major and career aspirations, but because the role of APCS course-taking must be understood in the context of which other variables were significant, we begin with an overview of such variables. Further, because we found a high degree of overlap in the variables significant in both the major and career regressions, the following section summarizes key findings across both models. In Table 1 (major) and Table 2 (career), significant main effects are shown in Model 1, whereas interaction effects are presented in Model 2. Given our large sample size, we only discuss variables significant at $p<.01$.

5.2.1 Significant Findings Across Major and Career Aspiration Regressions. Across both models, the likelihood of planning to pursue a computing major or career was greater among students who identified as men, Asian, Black, Hispanic, or multi-racial. Coming from a lower-income family or having at least one parent with a STEM career also predicted an increased interest in computing majors and careers. Computing aspirations were significantly lower among students who intended 
Table 1. Logistic Regression Predicting Intent to Major in CS Versus All Other Majors $(n=129,241)$

\begin{tabular}{|c|c|c|c|c|c|c|c|c|}
\hline \multirow[b]{2}{*}{ Variable } & \multicolumn{4}{|c|}{ Model 1} & \multicolumn{4}{|c|}{ Model 2} \\
\hline & $\mathrm{b}$ & & SE & $\operatorname{Exp}(B)$ & $\mathrm{b}$ & & $\mathrm{SE}$ & $\operatorname{Exp}(B)$ \\
\hline \multicolumn{9}{|l|}{$\overline{\text { Person Inputs }}$} \\
\hline Sex: Female & -0.728 & ** & 0.036 & 0.483 & -0.903 & ** & 0.042 & 0.405 \\
\hline \multicolumn{9}{|l|}{ Race/Ethnicity (vs. White) } \\
\hline American Indian & -0.269 & & 0.464 & 0.764 & -0.337 & & 0.526 & 0.714 \\
\hline Asian & 0.744 & ** & 0.041 & 2.105 & 0.939 & ** & 0.049 & 2.556 \\
\hline Black & 0.647 & ** & 0.062 & 1.909 & 0.654 & ** & 0.068 & 1.924 \\
\hline Hispanic & 0.371 & ** & 0.066 & 1.449 & 0.377 & ** & 0.072 & 1.459 \\
\hline Two or more races & 0.173 & ** & 0.053 & 1.189 & 0.244 & ** & 0.063 & 1.277 \\
\hline Another race/ethnicity & 0.397 & & 0.177 & 1.488 & 0.403 & ** & 0.179 & 1.496 \\
\hline $\begin{array}{l}\text { Understanding of computer } \\
\text { programming is important }\end{array}$ & 0.089 & ** & 0.017 & 1.093 & 0.089 & ** & 0.017 & 1.093 \\
\hline \multicolumn{9}{|l|}{ Background Contextual Affordances } \\
\hline Family income & -0.104 & ** & 0.020 & 0.901 & -0.101 & ** & 0.020 & 0.904 \\
\hline First-generation college student & 0.053 & & 0.050 & 1.054 & 0.056 & & 0.050 & 1.057 \\
\hline $\begin{array}{l}\text { At least one parent in a STEM } \\
\text { career }\end{array}$ & 0.103 & * & 0.034 & 1.108 & 0.104 & * & 0.034 & 1.109 \\
\hline \multicolumn{9}{|l|}{ Learning Experiences } \\
\hline High school GPA & 0.033 & & 0.020 & 1.033 & 0.035 & & 0.020 & 1.036 \\
\hline $\begin{array}{l}\text { Number of non-CS AP } \\
\text { math/science courses taken }\end{array}$ & -0.152 & ** & 0.019 & 0.859 & -0.143 & ** & 0.018 & 0.867 \\
\hline Took only APCSA & 0.321 & ** & 0.047 & 1.379 & 0.367 & ** & 0.064 & 1.444 \\
\hline Took only APCSP & 0.151 & & 0.076 & 1.163 & 0.107 & & 0.117 & 1.113 \\
\hline Took both APCS courses & 0.638 & ** & 0.090 & 1.893 & 0.701 & ** & 0.130 & 2.016 \\
\hline $\begin{array}{l}\text { In past year: Written computer } \\
\text { code }\end{array}$ & 0.517 & ** & 0.015 & 1.676 & 0.505 & ** & 0.015 & 1.657 \\
\hline Composite ACT score & 0.163 & ** & 0.028 & 1.177 & 0.159 & ** & 0.028 & 1.173 \\
\hline \multicolumn{9}{|l|}{ Self-Efficacy } \\
\hline $\begin{array}{l}\text { Self-rated: Computer programming } \\
\text { skills }\end{array}$ & 0.666 & ** & 0.020 & 1.947 & 0.672 & ** & 0.020 & 1.957 \\
\hline Academic Self-Concept ${ }^{\mathrm{a}}$ & -0.021 & & 0.022 & 0.979 & -0.021 & ** & 0.022 & 0.979 \\
\hline Social Self-Concept ${ }^{\mathrm{a}}$ & -0.219 & ** & 0.019 & 0.803 & -0.221 & ** & 0.019 & 0.802 \\
\hline Science Self-Efficacy ${ }^{\mathrm{a}}$ & -0.077 & ** & 0.022 & 0.926 & -0.080 & ** & 0.022 & 0.923 \\
\hline Science Identity ${ }^{\mathrm{a}}$ & 0.203 & ** & 0.021 & 1.225 & 0.198 & ** & 0.021 & 1.219 \\
\hline Pluralistic Orientation $^{\mathrm{a}}$ & 0.039 & & 0.018 & 1.039 & 0.041 & ** & 0.018 & 1.042 \\
\hline Habits of Mind ${ }^{\mathrm{a}}$ & -0.059 & * & 0.020 & 0.943 & -0.059 & * & 0.020 & 0.943 \\
\hline \multicolumn{9}{|l|}{ Outcome Expectations } \\
\hline Likelihood of staying in major field & 0.093 & ** & 0.018 & 1.098 & 0.095 & ** & 0.018 & 1.100 \\
\hline \multicolumn{9}{|l|}{ Interests } \\
\hline $\begin{array}{l}\text { Attending college to be able to get } \\
\text { a better job }\end{array}$ & 0.012 & & 0.019 & 1.012 & 0.013 & & 0.019 & 1.013 \\
\hline $\begin{array}{l}\text { Attending college to be able to } \\
\text { make more money }\end{array}$ & 0.109 & ** & 0.020 & 1.115 & 0.105 & ** & 0.020 & 1.111 \\
\hline $\begin{array}{l}\text { Attending college to get training } \\
\text { for a specific career }\end{array}$ & 0.077 & ** & 0.018 & 1.081 & 0.077 & ** & 0.018 & 1.080 \\
\hline
\end{tabular}


Table 1. Continued

\begin{tabular}{|c|c|c|c|c|c|c|c|c|}
\hline \multirow[b]{2}{*}{ Variable } & \multicolumn{4}{|c|}{ Model 1} & \multicolumn{4}{|c|}{ Model 2} \\
\hline & $\mathrm{b}$ & & SE & $\operatorname{Exp}(B)$ & $\mathrm{b}$ & & SE & $\overline{\operatorname{Exp}(\mathrm{B})}$ \\
\hline $\begin{array}{l}\text { Attending college to gain a general } \\
\text { education and appreciation of ideas }\end{array}$ & -0.015 & & 0.015 & 0.985 & -0.014 & & 0.015 & 0.986 \\
\hline Social Agency ${ }^{\mathrm{a}}$ & -0.075 & ** & 0.021 & 0.928 & -0.079 & ** & 0.021 & 0.924 \\
\hline Artist Personality ${ }^{\mathrm{a}}$ & -0.114 & ** & 0.019 & 0.892 & -0.114 & ** & 0.019 & 0.892 \\
\hline Striver Personality ${ }^{\mathrm{a}}$ & -0.041 & & 0.020 & 0.959 & -0.040 & & 0.020 & 0.961 \\
\hline Goal: Raising a family & -0.079 & ** & 0.017 & 0.924 & -0.073 & ** & 0.018 & 0.930 \\
\hline \multicolumn{9}{|l|}{ Contextual Influences } \\
\hline Selectivity of institution & 0.103 & ** & 0.024 & 1.109 & 0.090 & ** & 0.024 & 1.094 \\
\hline $\begin{array}{l}\text { Institution type: Four-year (vs. } \\
\text { university) }\end{array}$ & 0.036 & & 0.042 & 1.036 & 0.033 & & 0.042 & 1.033 \\
\hline $\begin{array}{l}\text { Institution control: Public (vs. } \\
\text { private) }\end{array}$ & 0.173 & ** & 0.037 & 1.189 & 0.181 & ** & 0.037 & 1.199 \\
\hline $\begin{array}{l}\text { Concern about ability to finance } \\
\text { college education }\end{array}$ & -0.030 & & 0.017 & 0.970 & -0.027 & & 0.017 & 0.973 \\
\hline \multicolumn{9}{|l|}{ Choice Goals } \\
\hline \multicolumn{9}{|l|}{$\underline{\text { Degree Aspirations (vs. BA) }}$} \\
\hline $\mathrm{PhD}$ & -1.024 & ** & 0.051 & 0.359 & -1.014 & ** & 0.051 & 0.363 \\
\hline Law & -1.286 & ** & 0.127 & 0.276 & -1.283 & ** & 0.127 & 0.277 \\
\hline Medical & -2.658 & ** & 0.118 & 0.070 & -2.647 & ** & 0.118 & 0.071 \\
\hline Master's/MDiv & -0.283 & ** & 0.037 & 0.753 & -0.282 & ** & 0.037 & 0.754 \\
\hline Other & -0.830 & ** & 0.202 & 0.436 & -0.822 & $* *$ & 0.200 & 0.440 \\
\hline \multicolumn{9}{|l|}{ Interaction Terms } \\
\hline Female x APCSA & & & & & 0.713 & ** & 0.087 & 2.040 \\
\hline Female x APCSP & & & & & 0.517 & ** & 0.155 & 1.677 \\
\hline Female x Both APCS courses & & & & & 0.768 & $* *$ & 0.197 & 2.156 \\
\hline American Indian x APCSA & & & & & 0.163 & & 1.686 & 1.176 \\
\hline American Indian x APCSP & & & & & -17.985 & & $40,192.9$ & 0 \\
\hline American Indian x Both APCS cours & & & & & 0.800 & & 2.000 & 0.689 \\
\hline Asian x APCSA & & & & & -0.583 & $* *$ & 0.087 & 0.558 \\
\hline Asian x APCSP & & & & & -0.393 & & 0.176 & 0.675 \\
\hline Asian $x$ Both APCS & & & & & -0.750 & ** & 0.196 & 0.472 \\
\hline Black x APCSA & & & & & 0.065 & & 0.194 & 1.067 \\
\hline Black x APCSP & & & & & 0.154 & & 0.301 & 1.167 \\
\hline Black x Both APCS & & & & & 0.061 & & 0.364 & 1.063 \\
\hline Hispanic x APCSA & & & & & 0.170 & & 0.195 & 1.185 \\
\hline Hispanic x APCSP & & & & & 0.228 & & 0.252 & 1.256 \\
\hline Hispanic x Both APCS & & & & & -0.504 & & 0.398 & 0.604 \\
\hline Two or more races $\mathrm{x}$ APCSA & & & & & -0.271 & & 0.132 & 0.763 \\
\hline Two or more races $\mathrm{x}$ APCSP & & & & & -0.298 & & 0.241 & 0.742 \\
\hline Two or more races $\mathrm{x}$ Both APCS cou & arses & & & & -0.089 & & 0.280 & 0.915 \\
\hline
\end{tabular}

$\mathrm{b}=$ regression coefficient in log-odds; $\mathrm{SE}=$ standard error of the coefficient; $\operatorname{Exp}(\mathrm{B})=$ odds ratios.

${ }^{a}$ Latent construct; see Appendix Table A.1.

${ }^{*} p<.01 .{ }^{* *} p<.001$. 
Table 2. Logistic Regression Predicting Career Aspirations in Computing and Technology Versus All Other Careers $(n=129,241)$

\begin{tabular}{|c|c|c|c|c|c|c|c|c|}
\hline \multirow[b]{2}{*}{ Variable } & \multicolumn{4}{|c|}{ Model 1} & \multicolumn{4}{|c|}{ Model 2} \\
\hline & $\mathrm{b}$ & & $\mathrm{SE}$ & $\overline{\operatorname{Exp}(B)}$ & $\mathrm{b}$ & & $\mathrm{SE}$ & $\overline{\operatorname{Exp}(B)}$ \\
\hline \multicolumn{9}{|l|}{ Person Inputs } \\
\hline Sex: Female & -0.809 & ** & 0.041 & 0.445 & -1.001 & ** & 0.048 & 0.368 \\
\hline \multicolumn{9}{|l|}{ Race/Ethnicity (vs. white) } \\
\hline American Indian & -0.292 & & 0.553 & 0.747 & -0.471 & & 0.695 & 0.625 \\
\hline Asian & 0.646 & ** & 0.045 & 1.908 & 0.820 & ** & 0.056 & 2.271 \\
\hline Black & 0.661 & ** & 0.069 & 1.938 & 0.697 & ** & 0.075 & 2.008 \\
\hline Hispanic & 0.375 & ** & 0.073 & 1.455 & 0.425 & ** & 0.080 & 1.529 \\
\hline Two or more races & 0.204 & ** & 0.058 & 1.226 & 0.279 & ** & 0.070 & 1.322 \\
\hline Another race/ethnicity & 0.383 & & 0.197 & 1.467 & 0.389 & & 0.199 & 1.476 \\
\hline $\begin{array}{l}\text { Understanding of computer } \\
\text { programming is important }\end{array}$ & 0.053 & * & 0.018 & 1.055 & 0.054 & * & 0.018 & 1.055 \\
\hline \multicolumn{9}{|l|}{ Background Contextual Affordances } \\
\hline Family income & -0.169 & ** & 0.023 & 0.844 & -0.166 & ** & 0.023 & 0.847 \\
\hline First-generation college student & 0.081 & & 0.055 & 1.085 & 0.084 & & 0.055 & 1.088 \\
\hline $\begin{array}{l}\text { At least one parent in a STEM } \\
\text { career }\end{array}$ & 0.296 & ** & 0.037 & 1.344 & 0.294 & ** & 0.037 & 1.342 \\
\hline \multicolumn{9}{|l|}{ Learning Experiences } \\
\hline High school GPA & 0.055 & & 0.022 & 1.057 & 0.058 & * & 0.022 & 1.060 \\
\hline $\begin{array}{l}\text { Number of non-CS AP } \\
\text { math/science courses taken }\end{array}$ & -0.158 & ** & 0.021 & 0.854 & -0.152 & ** & 0.020 & 0.859 \\
\hline Took only APCSA & 0.238 & * & 0.051 & 1.269 & 0.306 & ** & 0.069 & 1.359 \\
\hline Took only APCSP & 0.181 & & 0.081 & 1.199 & 0.107 & & 0.125 & 1.186 \\
\hline Took both APCS courses & 0.520 & ** & 0.094 & 1.682 & 0.457 & * & 0.136 & 1.580 \\
\hline $\begin{array}{l}\text { In past year: Written computer } \\
\text { code }\end{array}$ & 0.508 & ** & 0.016 & 1.662 & 0.497 & ** & 0.016 & 1.644 \\
\hline Composite ACT score & 0.160 & ** & 0.031 & 1.174 & 0.158 & ** & 0.031 & 1.171 \\
\hline \multicolumn{9}{|l|}{ Self-Efficacy } \\
\hline $\begin{array}{l}\text { Self-rated: Computer programming } \\
\text { skills }\end{array}$ & 0.665 & ** & 0.023 & 1.945 & 0.668 & ** & 0.023 & 1.950 \\
\hline Academic Self-Concept ${ }^{\mathrm{a}}$ & -0.052 & & 0.024 & 0.949 & -0.053 & & 0.024 & 0.948 \\
\hline Social Self-Concept ${ }^{\mathrm{a}}$ & -0.251 & ** & 0.021 & 0.778 & -0.253 & ** & 0.021 & 0.776 \\
\hline Science Self-Efficacy ${ }^{a}$ & -0.090 & ** & 0.024 & 0.914 & -0.092 & ** & 0.024 & 0.912 \\
\hline Science Identity ${ }^{\mathrm{a}}$ & 0.249 & ** & 0.024 & 1.283 & 0.244 & ** & 0.024 & 1.277 \\
\hline Pluralistic Orientation $^{\mathrm{a}}$ & 0.066 & * & 0.020 & 1.068 & 0.069 & * & 0.020 & 1.071 \\
\hline Habits of Mind ${ }^{\mathrm{a}}$ & -0.047 & & 0.022 & 0.954 & -0.047 & & 0.022 & 0.954 \\
\hline \multicolumn{9}{|l|}{ Outcome Expectations } \\
\hline $\begin{array}{l}\text { Likelihood of maintaining career } \\
\text { choice }\end{array}$ & 0.017 & & 0.019 & 1.017 & 0.021 & & 0.019 & 1.021 \\
\hline \multicolumn{9}{|l|}{ Interests } \\
\hline $\begin{array}{l}\text { Attending college to be able to get } \\
\text { a better job }\end{array}$ & 0.039 & & 0.022 & 1.040 & 0.040 & & 0.022 & 1.041 \\
\hline $\begin{array}{l}\text { Attending college to be able to } \\
\text { make more money }\end{array}$ & 0.102 & ** & 0.020 & 1.108 & 0.100 & ** & 0.022 & 1.105 \\
\hline
\end{tabular}


Table 2. Continued

\begin{tabular}{|c|c|c|c|c|c|c|c|c|}
\hline \multirow[b]{2}{*}{ Variable } & \multicolumn{4}{|c|}{ Model 1} & \multicolumn{4}{|c|}{ Model 2} \\
\hline & $\mathrm{b}$ & & SE & $\operatorname{Exp}(B)$ & $\mathrm{b}$ & & SE & $\overline{\operatorname{Exp}(\mathrm{B})}$ \\
\hline $\begin{array}{l}\text { Attending college to get training } \\
\text { for a specific career }\end{array}$ & 0.162 & ** & 0.020 & 1.176 & 0.161 & ** & 0.020 & 1.174 \\
\hline $\begin{array}{l}\text { Attending college to gain a general } \\
\text { education and appreciation of ideas }\end{array}$ & -0.032 & & 0.016 & 0.969 & -0.031 & & 0.016 & 0.969 \\
\hline Social Agency ${ }^{\mathrm{a}}$ & -0.089 & ** & 0.023 & 0.915 & -0.093 & ** & 0.023 & 0.911 \\
\hline Artist Personality ${ }^{\mathrm{a}}$ & -0.136 & ** & 0.021 & 0.873 & -0.136 & ** & 0.021 & 0.872 \\
\hline Striver Personality ${ }^{\mathrm{a}}$ & -0.055 & & 0.022 & 0.946 & -0.053 & & 0.022 & 0.948 \\
\hline Goal: Raising a family & -0.093 & ** & 0.019 & 0.911 & -0.089 & ** & 0.019 & 0.915 \\
\hline \multicolumn{9}{|l|}{ Contextual Influences } \\
\hline Selectivity of institution & 0.108 & ** & 0.027 & 1.114 & 0.096 & ** & 0.027 & 1.101 \\
\hline $\begin{array}{l}\text { Institution type: Four-year (vs. } \\
\text { university) }\end{array}$ & 0.019 & & 0.047 & 1.019 & 0.016 & & 0.047 & 1.016 \\
\hline $\begin{array}{l}\text { Institution control: Public (vs. } \\
\text { private) }\end{array}$ & 0.311 & ** & 0.041 & 1.365 & 0.318 & ** & 0.041 & 1.374 \\
\hline $\begin{array}{l}\text { Concern about ability to finance } \\
\text { college education }\end{array}$ & -0.031 & & 0.019 & 0.970 & -0.028 & & 0.019 & 0.973 \\
\hline \multicolumn{9}{|l|}{ Choice Goals } \\
\hline \multicolumn{9}{|l|}{ Degree Aspirations (vs. BA) } \\
\hline $\mathrm{PhD}$ & -0.988 & ** & 0.057 & 0.372 & -0.978 & ** & 0.056 & 0.376 \\
\hline Law & -1.873 & ** & 0.197 & 0.154 & -1.870 & ** & 0.196 & 0.154 \\
\hline Medical & -2.429 & ** & 0.123 & 0.088 & -2.424 & ** & 0.123 & 0.089 \\
\hline Master's/MDiv & -0.242 & ** & 0.041 & 0.785 & -0.242 & ** & 0.041 & 0.785 \\
\hline Other & -0.863 & ** & 0.233 & 0.422 & -0.847 & ** & 0.232 & 0.429 \\
\hline \multicolumn{9}{|l|}{ Interaction Terms } \\
\hline Female x APCSA & & & & & 0.742 & ** & 0.095 & 2.101 \\
\hline Female x APCSP & & & & & 0.481 & ** & 0.170 & 1.617 \\
\hline Female x Both APCS courses & & & & & 0.849 & ** & 0.211 & 2.337 \\
\hline American Indian x APCSA & & & & & 0.594 & & 1.784 & 1.811 \\
\hline American Indian x APCSP & & & & & -17.461 & & $40,192.9$ & 0 \\
\hline American Indian $x$ Both APCS cours & & & & & 1.301 & & 2.108 & 3.674 \\
\hline Asian x APCSA & & & & & -0.555 & ** & 0.094 & 0.574 \\
\hline Asian x APCSP & & & & & -0.269 & & 0.188 & 0.764 \\
\hline Asian $x$ Both APCS & & & & & -0.333 & ** & 0.204 & 0.716 \\
\hline Black x APCSA & & & & & -0.128 & & 0.209 & 0.880 \\
\hline Black x APCSP & & & & & 0.170 & & 0.317 & 1.185 \\
\hline Black x Both APCS & & & & & -0.282 & & 0.398 & 0.754 \\
\hline Hispanic x APCSA & & & & & -0.127 & & 0.215 & 0.881 \\
\hline Hispanic x APCSP & & & & & -0.006 & & 0.278 & 0.994 \\
\hline Hispanic $x$ Both APCS & & & & & -0.338 & & 0.427 & 0.713 \\
\hline Two or more races $\mathrm{x}$ APCSA & & & & & -0.248 & & 0.141 & 0.780 \\
\hline Two or more races $\mathrm{x}$ APCSP & & & & & -0.497 & & 0.269 & 0.609 \\
\hline Two or more races $x$ Both APCS cou & irses & & & & 0.080 & & 0.297 & 1.083 \\
\hline
\end{tabular}

$\mathrm{b}=$ regression coefficient in log-odds; $\mathrm{SE}=$ standard error of the coefficient; $\operatorname{Exp}(\mathrm{B})=$ odds ratios.

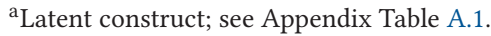

${ }^{*} p<.01 .{ }^{* *} p<.001$. 
to earn graduate/professional degrees, reported higher social self-concept, or who held stronger values and orientations around social agency, the arts, or family. Goals and values that were more predictive of aspirations for computing majors and careers included attending college with the goal of making more money or to get training for a specific career. Not surprisingly, many of these significant variables aligned with findings in prior research exploring CS major aspirations using similar TFS data [56].

Most of the additional variables that were significant across both regressions represented aspects of learning experiences and self-efficacy that were not available in the prior study using these data [56] but relate specifically to the computing and STEM domain. For example, reporting higher self-ratings of computing skills or having written computer code frequently in the past year predicted greater interest in computing majors and careers. More broadly, results revealed greater interest in computing majors and careers among students who reported higher levels of science identity but lower levels of science self-efficacy. In addition, greater numbers of AP math and AP science courses taken-other than APCS-negatively predicted interest in computing majors and careers, suggesting that students who take a broader range of advanced STEM courses in high school are less inclined to pursue computing majors and careers.

We now turn to the main focus of the present study, which is the extent to which taking APCS courses predicts first-year college students' major or probable career, above and beyond the effects associated with other variables in the model. Further, we examined how gender or race/ethnicity moderated the relationships between taking one or both APCS courses and students' interest in computing majors and careers.

5.2.2 APCS Course-Taking Predicting Computing Major. The logistic regression predicting intent to major in computing (see Table 1) revealed differences in the salience of our three APCS variables (taking only APCSA, only APCSP, or both APCS courses). As shown in the main effects model (Model 1), students who took only APCSA were 38\% more likely to indicate CS as their probable major (odds ratio: 1.379) than those who took no APCS courses, even when controlling for other relevant blocks of variables (Person Inputs, Background Contextual Affordances, Learning Experiences, etc.). Similarly, students who took both APCSA and APCSP were $89 \%$ more likely (odds ratio: 1.893) than students who took no APCS courses to indicate intent to major in CS. However, taking only APCSP was not a significant predictor of intent to major in CS.

5.2.2.1 Major by Gender Interactions. After running the main effects model, we tested whether relationships between taking APCS courses and majoring in computing were moderated by students' gender, seeking here to understand if the predictive power of APCS courses might differ between women and men. As shown in Model 2 of Table 1, all three interactions were significant. In other words, taking APCSA, APCSP, or both APCS courses were all more positively associated with women's aspirations to major in computing than they were for men. Notably, given that taking only APCSP was not significant in the main effects model, the interaction with gender suggests that taking the course was uniquely associated with women's intent to major in computing.

5.2.2.2 Major by Race/Ethnicity Interactions. As shown in Model 2 of Table 1, there were two significant interactions between APCS course-taking and students' racial and ethnic identities. Although taking only APCSA was a positive predictor of students' intentions to pursue a CS major for all students generally, the significant interaction term indicated that APCSA was a more salient predictor of majoring in computing for white students than for Asian students. We also found that taking both APCS courses was a stronger positive predictor of pursuing a major in CS for white students than for Asian students. 
5.2.3 APCS Course-Taking Predicting Computing or Technology Career Aspirations. Using the same independent variables as in the preceding analysis of major aspirations, we ran two models to predict students' aspirations to work in computing or technology fields versus all other career options (see Table 2). Similar to the findings predicting students' intent to major in computing, Model 1 shows that taking only APCSA or both APCS courses positively predicted students' interest in a computing career (although less so than these courses predicted students' interest in a computing major). As shown in Table 2, students who took only APCSA were 27\% more likely to indicate a probable career in computing (odds ratio: 1.269) than students who did not take any APCS courses. Students who took both APCSA and APCSP were 68\% more likely (odds ratio: 1.682) than students who did not take any APCS courses to indicate aspirations to work in computing and technology. Notably, taking only APCSP did not significantly predict students' interest in pursuing a computing career.

5.2.3.1 Career by Gender Interactions. We also explored whether the association between APCS course-taking and students' career aspirations was moderated by students' gender. We ran three cross-product interactions between each APCS course-taking variable and the gender variable. As shown in Model 2 of Table 2, all three interactions were significant. In other words, taking APCSA, APCSP, and both APCS courses appeared to have a stronger positive association with women's computing career aspirations than with those of men. Although the main effect of taking only APCSP was not significant in predicting computing career aspirations, the significant interaction term suggests that APCSP may play a role in encouraging computing career interests for women in particular.

5.2.3.2 Career by Race/Ethnicity Interactions. We also used interaction terms to explore whether the predictive power of taking any of the APCS course offerings on computing career aspirations was moderated by students' race or ethnicity. As seen in Table 2, only one of the interaction terms emerged as significant in predicting a student's computing career aspirations. Specifically, the positive association between taking only APCSA and students' aspirations to work in computing and tech was stronger among white students than among Asian students.

\section{DISCUSSION}

Interpretations of these findings depend on how one perceives the purpose of APCS courses. If these courses are viewed strictly as the primary entry point in the computing pipeline, scholars have already found that the addition of APCSP appears to have contributed to broadening participation by increasing the number of students who are taking at least one APCS course [13]. However, our study examined first-year college students' longer-term commitment to computing by assessing whether APCS course-taking was associated with aspirations to pursue degrees or careers in computing fields. After controlling for the predictive power of nearly three dozen variables reflecting different facets of SCCT, the results revealed that taking the traditional APCSA course, whether alone or in addition to APCSP, was associated with an increased likelihood of planning to major in or pursue careers in computing. However, taking only APCSP-although positively correlated with computing major and career choice based on the descriptive analysis-did not remain a significant predictor of these outcomes once the full range of independent variables was controlled.

Our findings did, however, suggest gender and racial/ethnic variations in the predictive power of APCS courses on students' computing majors and career intentions. For example, taking any of these courses (APCSA, APCSP, or both APCS courses) was more strongly associated with women's plans to pursue computing majors and careers than was the case for men. The finding regarding APCSP is especially noteworthy, as taking only APCSP was not predictive of computing 
intentions for our full sample. Thus, it is possible that the experience of taking APCS coursesincluding APCSP-uniquely encourages high school girls to consider computing pathways. Although causality cannot be determined from these data, it is worth noting that the College Board [68] reported a similar finding with respect to gender, albeit with a more limited set of control variables. Further, our race/ethnicity interactions revealed that taking APCSA (whether alone or in addition to APCSP) related more strongly to longer-term computing intentions for white students that for Asian students. Taking APCSP on its own, however, did not yield any differences in predictive power by students' race/ethnicity. In other words, across all racial/ethnic groups, computing major and career aspirations appeared unrelated to APCSP course-taking when the full range of variables were controlled.

\section{LIMITATIONS}

It is important to acknowledge several limitations to this study. First, our sample was restricted to first-time, full-time undergraduate students attending the 168 four-year colleges and universities that participated in the 2017 TFS. Therefore, findings may not reflect the experience of APCS course-takers who attended community colleges or other non-participating institutions, who were enrolled part-time, or who did not enter college in fall 2017. Our analyses would have benefited from a more representative sample of institutions that included two-year colleges, as community colleges can be a critical site of preparation for students who eventually earn degrees in computing $[8,37]$. Further, only a small number (9\%) of U.S. public or private high schools even offered APCSP in the 2016-2017 school year, narrowing the sample of students [16].

Second, our data were cross sectional, such that information on students' prior APCS coursetaking was collected at the same time as their major and career intentions were measured. Therefore, we cannot say whether or to what extent the APCS courses led to students' interests in computing majors or careers. Indeed, it is likely that prior interest in computing led certain students to enroll in APCS. As such, despite the number of control variables included in this study, we could not account for self-selection bias and causality could not be determined. In addition, the crosssectional nature of the data means that career and major aspirations were measured only at the point of college entry, despite the reality that students' interest in pursuing computing fields may change significantly during college $[33,55]$. Relatedly, this study did not account for the fact that many students who pursue non-computing majors and careers actually incorporate computing concepts in their chosen fields (bioinformatics, geoinformatics, etc.); this level of nuance could not be gleaned from the data used in this study.

Third, the sample only reflected students who had taken APCS courses prior to entering college in fall 2017. This time frame represents just 1 year after the official roll-out of APCSP in 20162017, although some students may have taken APCSP even earlier during the 2-year pilot phase. Thus, this study reflects the early stages of the APCSP course and does not account for changes that may have occurred in course content or delivery as more schools have adopted the course, as enrollments have continued to grow, and as instructors may have evolved their pedagogical approaches. We also do not know from these data the specific year in which students enrolled in APCS courses, nor which course they took first if they took both APCSA and APCSP.

A final set of limitations relates to the variables that were or were not included in our study. First, not all elements of SCCT are represented by the variables we included. Second, although SCCT has been widely used as a career development framework, we might also question whether it adequately speaks to the experiences and pathways of all students, especially those who do not follow prescriptive and normative school-to-career pathways. Including other variables into our model or using a different framework might have yielded contrasting results. Finally, our use of a relatively large number of control variables suggests that our regression results may be a 
conservative estimate of the association between APCSP course-taking and computing major and career intentions. In particular, we suspect that the distinction between our study's findings and that of the College Board [68] (as it relates to the predictive power of APCSP) results from our inclusion of a larger range of covariates, including students' computing experiences and self-efficacy.

\section{DIRECTIONS FOR FUTURE RESEARCH}

The limitations described previously point the way to important new directions for future research on the role of APCS courses and how students' interests in computing majors and careers develop. First, it is important to engage in research that deepens our understanding of what leads high school teachers and advisors to recommend that students take one APCS course over another, and specifically to learn whether the current two-course APCS structure might, perhaps unintentionally, cause some students to be "tracked" to a particular APCS course based on assumptions of ability or course rigor. If APCSA portends longer-term retention in the computing pipeline, more so than APCSP, analyses of who takes which APCS course when both are offered (and which course they take first) would help us consider the extent to which inequities are being reproduced in practice. This is especially important in light of the fact that taking APCSP positively correlates with computing aspirations among women, but it does not appear to boost computing major or career interests among students of color in particular.

With respect to students taking both APCS courses, this study showed that they had a much higher likelihood of intending to pursue computing majors and careers than students taking either APCSA or APCSP alone. The recent College Board report [68] conveys a similar finding. We believe that a deeper understanding of students who take both courses would add significant value to BPC efforts. Although both courses are considered introductory computing classes-and some assume that APCSP serves as a precursor to the more programming-focused APCSA course-it is possible for students to enroll in the two classes concurrently, something that ambitious collegegoing aspirants with access to both courses might elect to do, for the aforementioned benefits of augmenting high school transcripts with AP courses.

Next, future research should examine how many and which groups of students take APCS courses in various settings and formats. Our preliminary analyses of TFS respondents' high school IDs showed that some students who reported having taken APCSP attended schools that did not offer the course, at least not as indicated by the College Board. ${ }^{4}$ Students may have taken APCSP online, at other schools, or as an independent study. Ideally, future research on APCS would account for variations in course format while also considering the characteristics of schools offering the course, as well as information on course instructors and their pedagogical approaches. Adding such nuance to a study like the present one could shift, or at least better contextualize, our conclusions about APCSP course-takers' career and major aspirations.

Importantly, scholars aimed at determining causal connections between APCS courses and student outcomes would benefit from data that better account for myriad reasons that students enroll in these courses. Although our models controlled for students' motivations for attending college and for their major/career choices, the TFS data did not include students' own reasons for enrolling in APCS courses. Possible reasons include a desire to gain valuable skills in their ultimate quest for computing degrees, develop an understanding of basic computing concepts, and/or learn about the broader role of computing in society. Students' motivations for enrolling may also be highly dependent upon advice of teachers, counselors, family members, or peers. Further examination into students' reasons for taking APCS courses, how these vary among students taking APCSA,

\footnotetext{
${ }^{4}$ This observation is based on supplemental analyses we conducted by matching the survey respondents' high school IDs with data provided to us by the College Board on which schools offered APCSP between fall 2013 and spring 2017.
} 
APCSP, or both courses, and how they differ by students' gender, race/ethnicity, and other traits such as family income, high school type (private vs. public), and academic achievement (e.g., high school GPA, number of AP courses taken) would enable researchers to better assess the effectiveness of these courses as part of broader diversification efforts in computing. Additionally, prior research $[50,51,61]$ has also suggested that once enrolled in an introductory computing course, students' motivations and aspirations to major or work in a computing field may change in response to course content and pedagogy. A better understanding of how and why these aspirations change and for whom may shed light on pedagogical approaches, activities, and content that can help maintain student interest.

Finally, research on the role of APCS courses would also ideally follow students' pathways beyond the point of college entry and consider students' use of computing concepts in their noncomputing majors and careers. In doing so, researchers might also consider outcomes beyond those frequently associated with the pipeline metaphor (i.e., major or career intentions), as well as include others, such as the desire to develop an appreciation of technology in society or develop confidence or self-efficacy in programming. Researchers might also think even further out of the box by examining the potential of APCS courses to encourage a sense of agency and empowerment among students, especially for historically minoritized populations [52].

\section{CONCLUSION}

APCSP represents an important effort by educators, the College Board, and technology industry leaders to broaden the diversity of high school students who understand foundational computing concepts, appreciate the role of technology in society, and ultimately may seek further education and careers in computing fields. Its introduction several years ago marked a critical acknowledgment that to diversify computing in the United States, CS instruction needs to extend beyond the primarily coding-based emphasis of the traditional APCSA course. This study sought to understand the extent to which having taken one or both these APCS courses is associated with entering college students' interest in computing majors and careers. Our findings suggested a significant correlation between APCSA and longer-term computing aspirations, but that taking APCSP on its own was not predictive of students' intentions to major or seek employment in computing, at least when we accounted for the full range of control variables. However, we did find that taking APCS courses was significantly more predictive of women's interests in computing-related majors and careers than those of men, and that APCSP did predict women's longer-term computing interests despite the fact that it was not significant for the full sample nor for students of color in particular.

Although the findings raise a number of questions for educators and the College Board regarding who takes which course and why, consideration of these courses ought not exist in a vacuum. Indeed, discussions on the role of APCS courses must consider that both systems of education and $\mathrm{CS}$ as a field reside within and replicate broader systems of oppression such as sexism, racism, and other forms of exclusion $[6,20,49,52]$ and that technological innovations made by computer scientists have further amplified racial hierarchies by hiding, accelerating, and deepening discrimination under a guise of neutrality [7,48]. As such, APCSP is not-and should not be expected to be-a panacea for the structural oppressions that persist in schooling and likely in computing courses. Further, the true value of APCSP and other diversity and inclusion efforts cannot be measured solely within the school-career computing pipeline paradigm. Thus, rather than considering APCS courses within a fixed and narrow vocational pathway, it is important to explore how the pipeline itself serves to exclude students, particularly students of color. Expansive efforts to reenvision and transform computing education environments in the United States and elsewhere where broadening participation in computing is a concern must attend to how all students can be better welcomed into and supported in computing courses. 


\section{A APPENDIX}

\section{A.1 Variable List and Coding}

Table A.1.

\begin{tabular}{|c|c|c|}
\hline Variable & Coding & $\%$ Missing \\
\hline \multicolumn{3}{|l|}{ Personal Characteristics } \\
\hline Female (Reference Category: Male) & Dichotomous: $0=$ "Male," $1=$ "Female & 0.2 \\
\hline \multicolumn{3}{|l|}{ Race/Ethnicity (Reference Category: White) } \\
\hline American Indian & Dichotomous: $0=$ "No," $1=$ "Yes" & 0.3 \\
\hline Asian & Dichotomous: $0=$ "No," $1=$ "Yes" & 0.3 \\
\hline Black & Dichotomous: $0=$ "No," $1=$ "Yes" & 0.3 \\
\hline Hispanic & Dichotomous: $0=$ "No," 1 = "Yes" & 0.3 \\
\hline Multiracial & Dichotomous: $0=$ "No," 1 = "Yes" & 0.3 \\
\hline Other & Dichotomous: $0=$ "No," $1=$ "Yes" & 0.3 \\
\hline $\begin{array}{l}\text { A basic understanding of computer } \\
\text { programming is important for nearly any } \\
\text { career today }\end{array}$ & $\begin{array}{l}\text { 4-point scale: } 1=\text { "Strongly Disagree" to } 4= \\
\text { "Strongly Agree" }\end{array}$ & 8.6 \\
\hline \multicolumn{3}{|l|}{ Background Characteristics } \\
\hline Family income & $\begin{array}{l}\text { 12-point scale: } 1=\text { "Less than } \$ 15,000 " \text { to } \\
12=" \$ 500,000 \text { or more" }\end{array}$ & 6.7 \\
\hline First-generation college student & Dichotomous: $0=$ "No," $1=$ "Yes" & 2.8 \\
\hline At least one parent in a STEM career & $\begin{array}{l}\text { Dichotomous: } 0=\text { No parent in STEM } \\
\text { career, } 1=\text { At least } 1 \text { parent in STEM } \\
\text { (physician, engineer, health professional, } \\
\text { nurse, research scientist, IT professional, or } \\
\text { secondary STEM teacher) }\end{array}$ & 0 \\
\hline \multicolumn{3}{|l|}{ Learning Experiences } \\
\hline High school GPA & $\begin{array}{l}\text { 5-point scale: } 1=\text { "B- or below" to } 5=\text { "A or } \\
\text { A+" }\end{array}$ & 0.6 \\
\hline $\begin{array}{l}\text { Number of non-CS AP math/science } \\
\text { courses taken }\end{array}$ & $\begin{array}{l}\text { 7-point scale: } 0=0 \text { other AP Math or } \\
\text { Science courses to } 6=6 \text { other AP Math or } \\
\text { Science courses }\end{array}$ & 0 \\
\hline Took only AP Computer Science A & Dichotomous: $0=$ "No," $1=$ "Yes" & 12.3 \\
\hline $\begin{array}{l}\text { Took only AP Computer Science } \\
\text { Principles }\end{array}$ & Dichotomous: $0=$ "No," 1 = "Yes" & 12.3 \\
\hline Took both AP Computer Science Courses & Dichotomous: $0=$ "No," 1 = "Yes" & 12.3 \\
\hline In past year: Written computer code & $\begin{array}{l}\text { 3-point scale: } 1=\text { "Not at all," } 2= \\
\text { "Occasionally," } 3=\text { "Frequently" }\end{array}$ & 2.4 \\
\hline ACT Composite Score ${ }^{a}$ & 36-point scale & 20.8 \\
\hline \multicolumn{3}{|l|}{ Self-Efficacy } \\
\hline Self-rated computer programming skills & $\begin{array}{l}\text { 5-point scale: } 1=\text { "Lowest } 10 \% " \text { to } 5= \\
\text { "Highest } 10 \% "\end{array}$ & 5.7 \\
\hline Academic Self-Concept ${ }^{\mathrm{b}}$ & Four-item construct & 5.3 \\
\hline Self-rating: Academic ability & $\begin{array}{l}\text { 5-point scale: } 1=\text { "Lowest } 10 \% " \text { to } 5= \\
\text { "Highest } 10 \% "\end{array}$ & \\
\hline Self-rating: Drive to achieve & $\begin{array}{l}\text { 5-point scale: } 1=\text { "Lowest } 10 \% " \text { to } 5= \\
\text { "Highest } 10 \% "\end{array}$ & \\
\hline
\end{tabular}




\begin{tabular}{|c|c|c|}
\hline Variable & Coding & $\%$ Missing \\
\hline Self-rating: Mathematic ability & $\begin{array}{l}\text { 5-point scale: } 1=\text { "Lowest } 10 \% " \text { to } 5= \\
\text { "Highest } 10 \% "\end{array}$ & \\
\hline Self-rating: Intellectual self-confidence & $\begin{array}{l}\text { 5-point scale: } 1=\text { "Lowest } 10 \% " \text { to } 5= \\
\text { "Highest } 10 \% "\end{array}$ & \\
\hline Social Self-Concept ${ }^{\mathrm{b}}$ & Three-item construct & 5.6 \\
\hline Self-rating: Leadership ability & $\begin{array}{l}\text { 5-point scale: } 1=\text { "Lowest } 10 \% " \text { to } 5= \\
\text { "Highest } 10 \% "\end{array}$ & \\
\hline Self-rating: Public speaking ability & $\begin{array}{l}\text { 5-point scale: } 1=\text { "Lowest } 10 \% " \text { to } 5= \\
\text { "Highest } 10 \% "\end{array}$ & \\
\hline Self-rating: Social self-confidence & $\begin{array}{l}\text { 5-point scale: } 1=\text { "Lowest } 10 \% " \text { to } 5= \\
\text { "Highest } 10 \% "\end{array}$ & \\
\hline Science Self-Efficacy ${ }^{b}$ & Ten-item construct & 4.0 \\
\hline $\begin{array}{l}\text { Can use technical science skills (use of } \\
\text { tools, instruments, and/or techniques) }\end{array}$ & $\begin{array}{l}\text { 5-point scale: } 1=\text { "Not at all" to } 5= \\
\text { "Absolutely" }\end{array}$ & \\
\hline Can generate a research question & $\begin{array}{l}\text { 5-point scale: } 1=\text { "Not at all" to } 5= \\
\text { "Absolutely" }\end{array}$ & \\
\hline $\begin{array}{l}\text { Can determine how to collect appropriate } \\
\text { data }\end{array}$ & $\begin{array}{l}\text { 5-point scale: } 1=\text { "Not at all" to } 5= \\
\text { "Absolutely" }\end{array}$ & \\
\hline Can explain the results of a study & $\begin{array}{l}\text { 5-point scale: } 1=\text { "Not at all" to } 5= \\
\text { "Absolutely" }\end{array}$ & \\
\hline $\begin{array}{l}\text { Can use scientific literature to guide } \\
\text { research }\end{array}$ & $\begin{array}{l}\text { 5-point scale: } 1=\text { "Not at all" to } 5= \\
\text { "Absolutely" }\end{array}$ & \\
\hline $\begin{array}{l}\text { Can integrate results from multiple } \\
\text { studies }\end{array}$ & $\begin{array}{l}\text { 5-point scale: } 1=\text { "Not at all" to } 5= \\
\text { "Absolutely" }\end{array}$ & \\
\hline Can ask relevant questions & $\begin{array}{l}\text { 5-point scale: } 1=\text { "Not at all" to } 5= \\
\text { "Absolutely" }\end{array}$ & \\
\hline $\begin{array}{l}\text { Can identify what is known and not } \\
\text { known about a problem }\end{array}$ & $\begin{array}{l}\text { 5-point scale: } 1=\text { "Not at all" to } 5= \\
\text { "Absolutely" }\end{array}$ & \\
\hline Can understand scientific concepts & $\begin{array}{l}\text { 5-point scale: } 1=\text { "Not at all" to } 5= \\
\text { "Absolutely" }\end{array}$ & \\
\hline $\begin{array}{l}\text { Can see connections between different } \\
\text { areas of science and mathematics }\end{array}$ & $\begin{array}{l}\text { 5-point scale: } 1=\text { "Not at all" to } 5= \\
\text { "Absolutely" }\end{array}$ & \\
\hline Science Identity ${ }^{\mathrm{b}}$ & Four-item construct & 11.0 \\
\hline $\begin{array}{l}\text { I have a strong sense of belonging to a } \\
\text { community of scientists }\end{array}$ & $\begin{array}{l}\text { 5-point scale: } 1=\text { "Strongly disagree" to } 5= \\
\text { "Strongly agree" }\end{array}$ & \\
\hline $\begin{array}{l}\text { I derive great personal satisfaction from } \\
\text { working on a team that is doing } \\
\text { important research }\end{array}$ & $\begin{array}{l}5 \text {-point scale: } 1=\text { "Strongly disagree" to } 5= \\
\text { "Strongly agree" }\end{array}$ & \\
\hline I think of myself as a scientist & $\begin{array}{l}5 \text {-point scale: } 1=\text { "Strongly disagree" to } 5= \\
\text { "Strongly agree" }\end{array}$ & \\
\hline I feel like I belong in the field of science & $\begin{array}{l}\text { 5-point scale: } 1=\text { "Strongly disagree" to } 5= \\
\text { "Strongly agree" }\end{array}$ & \\
\hline Pluralistic Orientation $^{\mathrm{b}}$ & Five-item construct & 2.2 \\
\hline $\begin{array}{l}\text { Self-rating: Ability to see the world from } \\
\text { someone else's perspective }\end{array}$ & $\begin{array}{l}\text { 5-point scale: } 1=\text { "A major weakness" to } 5 \\
=\text { "A major strength" }\end{array}$ & \\
\hline
\end{tabular}




\begin{tabular}{|c|c|c|}
\hline Variable & Coding & $\%$ Missing \\
\hline $\begin{array}{l}\text { Self-rating: Tolerance of others with } \\
\text { different beliefs }\end{array}$ & $\begin{array}{l}\text { 5-point scale: } 1 \text { = "A major weakness" to } 5 \\
=\text { "A major strength" }\end{array}$ & \\
\hline $\begin{array}{l}\text { Self-rating: Openness to having my own } \\
\text { views challenged }\end{array}$ & $\begin{array}{l}\text { 5-point scale: } 1 \text { = "A major weakness" to } 5 \\
=\text { "A major strength" }\end{array}$ & \\
\hline $\begin{array}{l}\text { Self-rating: Ability to discuss and } \\
\text { negotiate controversial issues }\end{array}$ & $\begin{array}{l}\text { 5-point scale: } 1=\text { "A major weakness" to } 5 \\
=\text { "A major strength" }\end{array}$ & \\
\hline $\begin{array}{l}\text { Self-rating: Ability to work cooperatively } \\
\text { with diverse people }\end{array}$ & $\begin{array}{l}\text { 5-point scale: } 1 \text { = "A major weakness" to } 5 \\
=\text { "A major strength" }\end{array}$ & \\
\hline Habits of Mind ${ }^{b}$ & Nine-item construct & 2.7 \\
\hline $\begin{array}{l}\text { Frequency in past year: Ask questions in } \\
\text { class }\end{array}$ & $\begin{array}{l}\text { 3-point scale: } 1=\text { "Not at all," } 2= \\
\text { "Occasionally," } 3=\text { = Frequently" }\end{array}$ & \\
\hline $\begin{array}{l}\text { Frequency in past year: Support your } \\
\text { opinions with a logical argument }\end{array}$ & $\begin{array}{l}\text { 3-point scale: } 1=\text { "Not at all," } 2= \\
\text { "Occasionally," } 3=\text { = Frequently" }\end{array}$ & \\
\hline $\begin{array}{l}\text { Frequency in past year: Seek solutions to } \\
\text { problems and explain them to others }\end{array}$ & $\begin{array}{l}\text { 3-point scale: } 1=\text { "Not at all," } 2= \\
\text { "Occasionally," } 3 \text { = "Frequently" }\end{array}$ & \\
\hline $\begin{array}{l}\text { Frequency in past year: Take a risk } \\
\text { because you feel you have more to gain }\end{array}$ & $\begin{array}{l}\text { 3-point scale: } 1=\text { "Not at all," } 2= \\
\text { "Occasionally," } 3=\text { "Frequently" }\end{array}$ & \\
\hline $\begin{array}{l}\text { Frequency in past year: Seek alternative } \\
\text { solutions to a problem }\end{array}$ & $\begin{array}{l}\text { 3-point scale: } 1=\text { "Not at all," } 2= \\
\text { "Occasionally," } 3=\text { "Frequently" }\end{array}$ & \\
\hline $\begin{array}{l}\text { Frequency in past year: Look up scientific } \\
\text { research articles and resources }\end{array}$ & $\begin{array}{l}\text { 3-point scale: } 1=\text { "Not at all," } 2= \\
\text { "Occasionally," } 3=\text { = Frequently" }\end{array}$ & \\
\hline $\begin{array}{l}\text { Frequency in past year: Explore topics on } \\
\text { your own, even though it was not } \\
\text { required for class }\end{array}$ & $\begin{array}{l}\text { 3-point scale: } 1=\text { "Not at all," } 2= \\
\text { "Occasionally," } 3=\text { "Frequently" }\end{array}$ & \\
\hline $\begin{array}{l}\text { Frequency in past year: Accept mistakes } \\
\text { as part of the learning process }\end{array}$ & $\begin{array}{l}\text { 3-point scale: } 1=\text { "Not at all," } 2= \\
\text { "Occasionally," } 3=\text { "Frequently" }\end{array}$ & \\
\hline $\begin{array}{l}\text { Frequency in past year: Take on a } \\
\text { challenge that scares you }\end{array}$ & $\begin{array}{l}\text { 3-point scale: } 1=\text { "Not at all," } 2= \\
\text { "Occasionally," } 3=\text { "Frequently" }\end{array}$ & \\
\hline \multicolumn{3}{|l|}{ Outcome Expectations } \\
\hline Likelihood of maintaining major choice & $\begin{array}{l}\text { 4-point scale: } 1=\text { "Very good chance" to } 4= \\
\text { "No chance" }\end{array}$ & 13.1 \\
\hline Likelihood of maintaining career choice & $\begin{array}{l}\text { 4-point scale: } 1=\text { "Very good chance" to } 4= \\
\text { "No chance" }\end{array}$ & 13.2 \\
\hline \multicolumn{3}{|l|}{ Interests } \\
\hline Reason to attend college: Get a better Job & $\begin{array}{l}\text { 3-point scale: } 1=\text { "Not Important," } 2= \\
\text { "Somewhat Important," } 3=\text { "Very } \\
\text { Important" }\end{array}$ & 4.6 \\
\hline $\begin{array}{l}\text { Reason to attend college: Make more } \\
\text { money }\end{array}$ & $\begin{array}{l}\text { 3-point scale: } 1=\text { "Not Important," } 2= \\
\text { "Somewhat Important," } 3=\text { "Very } \\
\text { Important" }\end{array}$ & 4.8 \\
\hline $\begin{array}{l}\text { Reason to attend college: Training for a } \\
\text { specific career }\end{array}$ & $\begin{array}{l}\text { 3-point scale: } 1=\text { "Not Important," } 2= \\
\text { "Somewhat Important," } 3=\text { ="Very } \\
\text { Important" }\end{array}$ & 4.9 \\
\hline $\begin{array}{l}\text { Reason to attend college: Gain general } \\
\text { education and appreciation of ideas }\end{array}$ & $\begin{array}{l}\text { 3-point scale: } 1 \text { = "Not Important," } 2= \\
\text { "Somewhat Important," } 3 \text { = "Very } \\
\text { Important" }\end{array}$ & 4.7 \\
\hline
\end{tabular}




\begin{tabular}{|c|c|c|}
\hline Variable & Coding & $\%$ Missing \\
\hline Social Agency & Six-item construct & 12.2 \\
\hline $\begin{array}{l}\text { Goal: Keeping up to date with political } \\
\text { affairs }\end{array}$ & $\begin{array}{l}\text { 4-point scale: } 1=\text { "Not important" to } 4= \\
\text { "Essential" }\end{array}$ & \\
\hline $\begin{array}{l}\text { Goal: Participating in a community action } \\
\text { program }\end{array}$ & $\begin{array}{l}\text { 4-point scale: } 1=\text { "Not important" to } 4= \\
\text { "Essential" }\end{array}$ & \\
\hline Goal: Influencing social values & $\begin{array}{l}\text { 4-point scale: } 1=\text { "Not important" to } 4= \\
\text { "Essential" }\end{array}$ & \\
\hline Goal: Becoming a community leader & $\begin{array}{l}\text { 4-point scale: } 1=\text { "Not important" to } 4= \\
\text { "Essential" }\end{array}$ & \\
\hline Goal: Helping others who are in difficulty & $\begin{array}{l}\text { 4-point scale: } 1=\text { "Not important" to } 4= \\
\text { "Essential" }\end{array}$ & \\
\hline $\begin{array}{l}\text { Goal: Helping to promote racial } \\
\text { understanding }\end{array}$ & $\begin{array}{l}\text { 4-point scale: } 1=\text { "Not important" to } 4= \\
\text { "Essential" }\end{array}$ & \\
\hline \multicolumn{3}{|l|}{ Contextual Influences } \\
\hline Artist Personality $(\alpha=.736)$ & Four-item construct & 13.8 \\
\hline $\begin{array}{l}\text { Goal: Becoming accomplished in one of } \\
\text { the performing arts }\end{array}$ & $\begin{array}{l}\text { 4-point scale: } 1=\text { "Not important" to } 4= \\
\text { "Essential" }\end{array}$ & \\
\hline Goal: Creating artistic work & $\begin{array}{l}\text { 4-point scale: } 1=\text { "Not important" to } 4= \\
\text { "Essential" }\end{array}$ & \\
\hline Goal: Writing original works & $\begin{array}{l}\text { 4-point scale: } 1=\text { "Not important" to } 4= \\
\text { "Essential" }\end{array}$ & \\
\hline Self-rating: Artistic ability & $\begin{array}{l}\text { 5-point scale: } 1=\text { "Lowest } 10 \% " \text { to } 5= \\
\text { "Highest } 10 \% "\end{array}$ & \\
\hline Striver Personality $(\alpha=.667)$ & Four-item construct & 13.6 \\
\hline Goal: Becoming an authority in my field & $\begin{array}{l}\text { 4-point scale: } 1=\text { "Not important" to } 4= \\
\text { "Essential" }\end{array}$ & \\
\hline $\begin{array}{l}\text { Goal: Becoming successful in a business } \\
\text { of my own }\end{array}$ & $\begin{array}{l}\text { 4-point scale: } 1=\text { "Not important" to } 4= \\
\text { "Essential" }\end{array}$ & \\
\hline Goal: Being very well off financially & $\begin{array}{l}\text { 4-point scale: } 1=\text { "Not important" to } 4= \\
\text { "Essential" }\end{array}$ & \\
\hline $\begin{array}{l}\text { Goal: Obtaining recognition from my } \\
\text { colleagues for contributions to my special } \\
\text { field }\end{array}$ & $\begin{array}{l}\text { 4-point scale: } 1=\text { "Not important" to } 4= \\
\text { "Essential" }\end{array}$ & \\
\hline Goal: Raising a family & $\begin{array}{l}\text { 4-point scale: } 1=\text { "Not important" to } 4= \\
\text { "Essential" }\end{array}$ & 12.5 \\
\hline Selectivity & $\begin{array}{l}\text { Mean score of entering freshmen on the } \\
\text { Verbal/Math portions of the SAT or } \\
\text { converted ACT }\end{array}$ & 0.3 \\
\hline Four-year (vs. university) & $\begin{array}{l}\text { Dichotomous: } 0=\text { University; } 1=\text { Four-year } \\
\text { college }\end{array}$ & 0 \\
\hline Public (vs. private) & Dichotomous: $0=$ Public, $1=$ Private & 0 \\
\hline $\begin{array}{l}\text { Concern about ability to finance college } \\
\text { education }\end{array}$ & $\begin{array}{l}\text { 3-point scale: } 1 \text { = "None," } 2 \text { = "Some," } 3= \\
\text { "Major" }\end{array}$ & 1.2 \\
\hline \multicolumn{3}{|l|}{ Choice Goals } \\
\hline \multicolumn{3}{|l|}{$\begin{array}{l}\text { Degree aspirations (Reference Category: } \\
\text { Bachelor's) }\end{array}$} \\
\hline $\mathrm{PhD}$ & Dichotomous: $0=$ All others, $1=\mathrm{PhD}$ & 0 \\
\hline
\end{tabular}




\begin{tabular}{|c|c|c|}
\hline Variable & Coding & $\%$ Missing \\
\hline Law & Dichotomous: $0=$ All others, $1=$ Law & 0 \\
\hline Medicine & Dichotomous: $0=$ All others, $1=$ Medicine & 0 \\
\hline Master's & Dichotomous: $0=$ All others, $1=$ Master's & 0 \\
\hline Other & Dichotomous: $0=$ All others, $1=$ Other & 0 \\
\hline Dependent Variables & & 0 \\
\hline Intent to major in computer science & $\begin{array}{l}\text { Dichotomous: } 0=\text { All others, } 1=\text { "Computer } \\
\text { science," "Computer/Management } \\
\text { information systems," or "Other } \\
\text { math/computer science" }\end{array}$ & 2.3 \\
\hline Intent to pursue a computing career & $\begin{array}{l}\text { Dichotomous: } 0=\text { All others, } 1=\text { "Computer } \\
\text { programmer/developer," "Computer } \\
\text { systems analyst," or "Web designer" }\end{array}$ & 5.5 \\
\hline
\end{tabular}

aSAT scores were converted to ACT Composite scores for students using 2017 SAT Concordance Tables retrieved from https://www.act.org/content/act/en/products-and-services/the-act/scores/act-sat-concordance.html.

${ }^{\mathrm{b}}$ These constructs were created and validated by HERI. See Sharkness et al. [60] for details on development and reliability.

\section{ACKNOWLEDGMENTS}

The authors wish to thank the Cooperative Institutional Research Program at UCLA's Higher Education Research Institute for providing The Freshman Survey data used in this study, as well as the student survey respondents who made this work possible. This study was supported by Pivotal Ventures.

\section{REFERENCES}

[1] Michael W. Apple. 1993. What post-modernists forget: Cultural capital and official knowledge. Curriculum Studies 1, 3 (1993), 301-316. DOI : https://doi.org/10.1080/0965975930010301

[2] Andrea C. Arpaci-Dusseau, Owen L. Astrachan, Dwight Barnett, and Matthew Bauer. 2013. Computer science principles: Analysis of a proposed advanced placement course. In Proceedings of the 44th ACM Technical Symposium on Computer Science Education. ACM, New York, NY, 251-256. DOI : https://doi.org/10.1145/2445196.2445273

[3] William Aspray. 2016. Race and Computing: A History of Efforts to Broaden Participation of African Americans, Hispanics, and American Indians in Computing in the United States. Springer, New York, NY.

[4] Albert Bandura. 1985. Social Foundations of Thought and Action: A Social Cognitive Theory. Prentice Hall, Englewood Cliffs, NJ.

[5] Eric R. Banilower, P. Sean Smith, Kristen A. Malzahn, Courtney L. Plumley, Evelyn M. Gordon, and Meredith L. Hayes. 2018. Report of the 2018 NSSME+. Horizon Research, Inc., Chapel Hill, NC.

[6] Maya A. Beasley. 2012. Opting Out: Losing the Potential of America's Young Black Elite. University of Chicago Press, Chicago, IL.

[7] Ruha Benjamin. 2020. Race after technology: Abolitionist tools for the New Jim Code. Social Forces 98, 4 (2020), 1-3. DOI : https://doi.org/10.1093/sf/soz162

[8] Jennifer M. Blaney. 2020. Broadening participation in computing: The role of upward transfer. In Proceedings of the 51st ACM Technical Symposium on Computer Science Education. ACM, New York, NY, 254-260. DOI : https://doi.org/ $10.1145 / 3328778.3366807$

[9] John J. Burke. 2014. Makerspaces: A Practical Guide for Librarians. Rowman \& Littlefield, Lanham, MD.

[10] James Carpenter and Michael Kenward. 2012. Multiple Imputation and Its Application. John Wiley \& Sons, Hoboken, NJ.

[11] Anthony Carpi, Darcy M. Ronan, Heather M. Falconer, and Nathan H. Lents. 2017. Cultivating minority scientists: Undergraduate research increases self-efficacy and career ambitions for underrepresented students in STEM. Fournal of Research in Science Teaching 54, 2 (2017), 169-194. DOI : https://doi.org/10.1002/tea.21341

[12] Hadi Partovi. 2018. Girls and minorities break records in computer science as fastest-growing groups! Medium. Retrieved February 4, 2021 from https://codeorg.medium.com/girls-and-minorities-break-records-in-computerscience-as-fastest-growing-groups-39d23425810e 
[13] Code.org. 2020. Dig deeper into AP computer science. Retrieved February 4, 2021 from https://code.org/promote/ap

[14] College Board. n.d. AP Around the World. Retrieved August 26, 2021 from https://apstudents.collegeboard.org/aparound-the-world

[15] College Board. 2018. AP Central: AP Computer Science Principles. Retrieved February 4, 2021 from https://apcentral collegeboard.org/courses/ap-computer-science-principles?course=ap-computer-science-principles

[16] College Board. 2017. States propel students to success in advanced placement. College Board Newsroom. Retrieved August 24, 2021 from https://newsroom.collegeboard.org/states-propel-students-success-advanced-placement

[17] Cooperative Institutional Research Program [CIRP]. 2017. CIRP Construct Technical Report: 2016-2017 Appendix Construct Parameters. Higher Education Research Institute at UCLA. Retrieved February 4, 2021 from https://www.heri. ucla.edu/PDFs/constructs/Appendix2017.pdf

[18] Jan Cuny. 2015. Transforming k-12 computing education: An update and a call to action. ACM Inroads 6, 3 (2015), 54-57. DOI : https://doi.org/10.1145/2809795

[19] Jan Cuny. 2015. Transforming K-12 computing education: $\mathrm{AP}{ }^{\circledR}$ computer science principles. ACM Inroads 6, 4 (2015), 58-59. DOI : https://doi.org/10.1145/2832916

[20] Kim-Mai Cutler. 2015. East of Palo Alto's Eden: Race and the formation of Silicon Valley. TechCrunch. Retrieved February 4, 2021 from https://techcrunch.com/2015/01/10/east-of-palo-altos-eden/

[21] Nadya A. Fouad and Mercedes C. Santana. 2017. SCCT and underrepresented populations in STEM fields: Moving the needle. Fournal of Career Assessment 25, 1 (2017), 24-39. DOI : https://doi.org/10.1177/1069072716658324

[22] Cary Funk and Kim Parker. 2018. Women and men in STEM often at odds over workplace equity. Pew Research Center. Retrieved February 4, 2021 from https://www.pewsocialtrends.org/2018/01/09/women-and-men-in-stem-often-atodds-over-workplace-equity/

[23] Joanna Goode. 2008. Increasing diversity in K-12 computer science: Strategies from the field. In Proceedings of the 39th ACM Technical Symposium on Computer Science Education. ACM, New York, NY, 362-366. DOI : https://doi.org/ $10.1145 / 1352135.1352259$.

[24] Joanna Goode and Jane Margolis. 2011. Exploring computer science: A case study of school reform. ACM Transactions on Computing Education 11, 2 (2011), 1-16. DOI: https://doi.org/10.1145/1993069.1993076

[25] Jessica Guynn. 2015. Code.org targets high school computer science. USA Today. Retrieved February 4, 2021 from https://www.usatoday.com/story/tech/2015/05/14/codeorg-college-board-computer-science-high-school-diversity/ 27304593/

[26] Keith E. Howard and Douglas D. Havard. 2019. Advanced placement (AP) computer science principles: Searching for equity in a two-tiered solution to underrepresentation. Fournal of Computer Science Integration 2, 1 (2019), 1-15. DOI : https://doi.org/10.26716/jcsi.2019.02.1.1

[27] Mercedes Inda, Carmen Rodríguez, and José Vicente Peña. 2013. Gender differences in applying social cognitive career theory in engineering students. Fournal of Vocational Behavior 83, 3 (2013), 346-355. DOI: https://doi.org/10. 1177/0894845315599253

[28] June P. John and Martin Carnoy. 2017. Race and Gender Trends in Computer Science in the Silicon Valley from 1980-2015. Stanford University Center for Education Policy Analysis, Palo Alto, CA.

[29] Lisa Kaczmarczyk and Renee Dopplick. 2014. Rebooting the Pathway to Success: Preparing Students for Computing Workforce Needs in the United States. ACM Educational Policy Committee, New York, NY.

[30] Myungsook Klassen, Russell Stockard, and Ali Akbari. 2004. Stimulating information technology education among underrepresented minorities. In Proceedings of the 5th Conference on Information Technology Education. ACM, New York, NY, 278-278. DOI : https://doi.org/10.1145/1029533.1029611

[31] Hang-Shim Lee, Lisa Y. Flores, Rachel L. Navarro, and Marlen Kanagui-Muñoz. 2015. A longitudinal test of social cognitive career theory's academic persistence model among Latino/a and white men and women engineering students. fournal of Vocational Behavior 88, (2015), 95-103. DOI : https://doi.org/10.1016/j.jvb.2015.02.003

[32] Kathleen J. Lehman, Kaitlin N. S. Newhouse, and Linda J. Sax. 2020. Global participation in undergraduate computing: A review and agenda for research. In The Wiley Handbook of Gender Equity in Higher Education, Nancy S. Niemi and Marcus B. Weaver-Hightower (eds.). Wiley, 289-313. DOI : https://doi.org/10.1002/9781119257639.ch15

[33] Kathleen J. Lehman, Annie M. Wofford, Michelle Sendowski, Kaitlin N. S. Newhouse, and Linda J. Sax. 2020. Better late than never: Exploring students' pathways to computing in later stages of college. In Proceedings of the 51st ACM Technical Symposium on Computer Science Education. ACM, New York, NY, 1075-1081. DOI : https://doi.org/10.1145/ 3328778.3366814

[34] Robert W. Lent, Steven D. Brown, and Gail Hackett. 1994. Toward a unifying social cognitive theory of career and academic interest, choice, and performance. Journal of Vocational Behavior 45, 1 (1994), 79-122. DOI : https://doi.org/ 10.1006/jvbe.1994.1027

[35] Robert W. Lent, Steven D. Brown, and Gail Hackett. 2000. Contextual supports and barriers to career choice: A social cognitive analysis. fournal of Counseling Psychology 47, 1 (2000), 36. DOI : https://doi.org/10.1037/0022-0167.47.1.36 
[36] Robert W. Lent, Antonio M. Lopez Jr., Frederick G. Lopez, and Hung-Bin Sheu. 2008. Social cognitive career theory and the prediction of interests and choice goals in the computing disciplines. Fournal of Vocational Behavior 73, 1 (2008), 52-62. DOI : https://doi.org/10.1016/j.jvb.2008.01.002

[37] Louise Ann Lyon and Jill Denner. 2017. Community colleges: A resource for increasing equity and inclusion in computer science education. Communications of the ACM 60, 12 (2017), 24-26. DOI : https://doi.org/10.1145/3152914

[38] J. H. Maloney, K. Peppler, Y. Kafai, M. Resnick, and N. Rusk. 2008. Programming by choice: Urban youth learning programming with scratch. ACM SIGCSE Bulletin 40, 1 (2008), 367-371. DOI : https://doi.org/10.1145/1352135.1352260

[39] Jane Margolis, Rachel Estrella, Joanna Goode, Jennifer Jellison Holme, and Kim Nao. 2017. Stuck in the Shallow End: Education, Race, and Computing. MIT Press, Cambridge, MA.

[40] Alexis Martin and Allison Scott. 2013. Engaging underrepresented students in computer science: Examining the effectiveness of a 5-week computer science course in the SMASH summer academy. In Proceedings of the 2103 International Conference on Frontiers in Education: Computer Science and Computer Engineering. 362-366. DOI : https: //www.smash.org/wp-content/uploads/2013/08/examining_the_effectiveness_of_a_5-week_computer_science_ course_in__smash-2.pdf

[41] Krista D. Mattern, Emily J. Shaw, and Maureen Ewing. 2011. Advanced Placement ${ }^{\circledR}$ Exam Participation: Is AP® Exam Participation and Performance Related to Choice of College Major? College Board, New York, NY.

[42] Heather Mendick, Maria Berge, and Anna Danielsson. 2017. A critique of the STEM pipeline: Young people's identities in Sweden and science education policy. British fournal of Educational Studies 65, 4 (2017), 481-497. DOI : https://doi. org/10.1080/00071005.2017.1300232

[43] Michele S. Moses, Kenneth R. Howe, and Tricia Niesz. 1999. The pipeline and student perceptions of schooling: Good news and bad news. Educational Policy 13, 4 (1999), 573-591. DOI : https://doi.org/10.1177/0895904899013004005

[44] National Academies of Sciences, Engineering, and Medicine. 2021. Cultivating Interest and Competencies in Computing: Authentic Experiences and Design Factors. National Academies of Sciences, Engineering, and Medicine, Washington, DC. DOI : https://doi.org/10.17226/25912

[45] National Center for Women and Information Technology. 2019. NCWIT Fact Sheet. Retrieved February 4, 2021 from https://www.ncwit.org/ncwit-fact-sheet

[46] National Science Foundation. 2014. College board launches new AP computer science principles course. News Release 14-168. Retrieved February 4, 2021 from https://www.nsf.gov/news/news_summ.jsp?cntn_id=133571

[47] Rachel L. Navarro, Lisa Y. Flores, Hang-Shim Lee, and Rebecca Gonzalez. 2014. Testing a longitudinal social cognitive model of intended persistence with engineering students across gender and race/ethnicity. fournal of Vocational Behavior 85, 1 (2014), 146-155. DOI : https://doi.org/10.1016/j.jvb.2014.05.007

[48] Safiya Umoja Noble. 2018. Algorithms of Oppression: How Search Engines Reinforce Racism. NYU Press, New York, NY.

[49] Margaret O'Mara. 2020. The Code: Silicon Valley and the Remaking of America. Penguin Books, London, UK.

[50] Markeya S. Peteranetz, Abraham E. Flanigan, Duane F. Shell, and Leen-Kiat Soh. 2016. Perceived instrumentality and career aspirations in CS1 courses: Change and relationships with achievement. In Proceedings of the 2016 ACM Conference on International Computing Education Research (ICER'16). ACM, New York, NY, 13-21. DOI: https://doi. org/10.1145/2960310.2960320

[51] Markeya S. Peteranetz, Abraham E. Flanigan, Duane F. Shell, and Leen-Kiat Soh. 2018. Career aspirations, perceived instrumentality, and achievement in undergraduate computer science courses. Contemporary Educational Psychology 53, (2018), 27-44. DOI : https://doi.org/10.1016/j.cedpsych.2018.01.006

[52] Jean J. Ryoo, Tiera Tanksley, Cynthia Estrada, and Jane Margolis. 2020. Take space, make space: How students use computer science to disrupt and resist marginalization in schools. Computer Science Education 30, 3 (2020), 337-361. DOI : https://doi.org/10.1080/08993408.2020.1805284

[53] Alpaslan Sahin, Adem Ekmekci, and Hersh C. Waxman. 2017. The relationships among high school STEM learning experiences, expectations, and mathematics and science efficacy and the likelihood of majoring in STEM in college. International Journal of Science Education 39, 11 (2017), 1549-1572. DOI : https://doi.org/10.1080/09500693.2017. 1341067

[54] Linda J. Sax. 2008. The Gender Gap in College: Maximizing the Developmental Potential of Women and Men. Jossey-Bass, San Francisco, CA.

[55] Linda J. Sax, Kari L. George, Annie M. Wofford, and Sarayu Sundar. 2019. The tech trajectory: Examining the role of college environments in enhancing a diverse pipeline to computing careers. Paper presented at the annual meeting of the Association for the Study of Higher Education (ASHE). Portland, OR.

[56] Linda J. Sax, Kathleen J. Lehman, Jerry A. Jacobs, M. Allison Kanny, Gloria Lim, Laura Monje-Paulson, and Hilary B. Zimmerman. 2017. Anatomy of an enduring gender gap: The evolution of women's participation in computer science. Fournal of Higher Education 88, 2 (2016), 258-293. DOI : https://doi.org/10.1080/00221546.2016.1257306

[57] Linda J. Sax, Kaitlin N. S. Newhouse, Joanna Goode, Max Skorodinsky, Tomoko M. Nakajima, and Michelle Sendowski. 2020. Does AP CS principles broaden participation in computing? An analysis of APCSA and APCSP participants. 
In Proceedings of the 51st ACM Technical Symposium on Computer Science Education. ACM, New York, NY, 542-548. DOI : https://doi.org/10.1145/3328778.3366826

[58] Allison Scott, Sonia Koshy, Meghana Rao, Laura Hinton, Julie Flapan, Alexis Martin, and Frieda McAlear. 2019. Computer Science in California's Schools: An Analysis of Access, Enrollment, and Equity. Kapor Center, Oakland, CA.

[59] Kimberly A. Scott and Steve Elliott. 2020. STEM diversity and inclusion efforts for women of color: A critique of the new labor system. International fournal of Gender, Science and Technology 11, 3 (2020), 374-382. DOI : http: //genderandset.open.ac.uk/index.php/genderandset/article/view/646

[60] Jessica Sharkness, Lisa DeAngelo, and John Pryor. 2010. CIRP Construct Technical Report. Higher Education Research Institute. UCLA, Los Angeles, CA.

[61] Duane F. Shell, Leen-Kiat Soh, Abraham E. Flanigan, and Markeya S. Peteranetz. 2016. Students' initial course motivation and their achievement and retention in college CS1 courses. In Proceedings of the 47th ACM Technical Symposium on Computing Science Education (SIGCSE'16). ACM, New York, NY, 639-644. DOI: https://doi.org/10.1145/2839509. 2844606

[62] Matthew Soldner, Heather Rowan-Kenyon, Karen Kurotsuchi Inkelas, Jason Garvey, and Claire Robbins. 2012. Supporting students' intentions to persist in STEM disciplines: The role of living-learning programs among other social-cognitive factors. Journal of Higher Education 83, 3 (2012), 311-336. DOI : https://doi.org/10.1080/00221546.2012. 11777246

[63] Daniel G. Solorzano and Armida Ornelas. 2002. A critical race analysis of advanced placement classes: A case of educational inequality. Journal of Latinos and Education 1, 4 (2002), 215-229. DOI:https://doi.org/10.1207/ S1532771XJLE0104_2

[64] Ellen Bara Stolzenberg, M. Kevin Eagan, Melissa C. Aragon, Natacha M. Cesar-Davis, Sidronio Jacobo, Victoria Couch, and Cecelia Rios-Aguilar. 2019. The American Freshman: National Norms Fall 2017. Higher Education Research Institute. UCLA, Los Angeles, CA.

[65] Katalin Szelényi, Nida Denson, and Karen Kurotsuchi Inkelas. 2013. Women in STEM majors and professional outcome expectations: The role of living-learning programs and other college environments. Research in Higher Education 54, 8 (2013), 851-873. DOI : 10.1007/s11162-013-9299-2

[66] Anna Vitores and Adriana Gil-Juárez. 2016. The trouble with 'women in computing': A critical examination of the deployment of research on the gender gap in computer science. fournal of Gender Studies 25, 6 (2016), 666-680. DOI : https://doi.org/10.1080/09589236.2015.1087309

[67] Xueli Wang. 2013. Why students choose STEM majors: Motivation, high school learning, and postsecondary context of support. American Educational Research fournal 50, 5 (2013), 1081-1121. D0I:https://doi.org/10.3102/ 0002831213488622

[68] Jeff Wyatt, Jing Feng, and Maureen Ewing. 2020. AP Computer Science Principles and the STEM and Computer Science Pipelines. College Board, New York, NY. Retrieved February 4, 2021 from https://apcentral.collegeboard.org/pdf/apcsp-and-stem-cs-pipelines.pdf?course=ap-computer-science-principles

Received March 2021; revised September 2021; accepted September 2021 